Penultimate Draft of a paper in press in Nonlinear Dynamics in Psychology and the Life Sciences, 2005

\title{
Nonlinear dynamical analysis of noisy time series.
}

\author{
Andrew Heathcote \& David Elliott \\ School of Behavioural Sciences \\ The University of Newcastle
}

Australia

\section{RUNNING HEAD: Analysis of chaos in noise}

Correspondence should be addressed to: Andrew Heathcote, Aviation Building, The University of Newcastle, University Drive, Callaghan, 2308, NSW, Australia.

Email: andrew.heathcote@ newcastle.edu.au Phone: 61-2-49215952 Fax: 61-2-49216906 
Penultimate Draft of a paper in press in Nonlinear Dynamics in Psychology and the Life Sciences, 2005

Empirical time series in the life sciences are often non-stationary and have small signat to-noise ratios, making it difficult to accurately detect and characterize dynamical structure. The usual response to high noise is averaging, but time domain averaging is inappropriate, especially when the dynamics are nonlinear. We review alternative delayspace averaging methods based on the topology and short-term predictability of nonlinear dynamics and illustrate their application using the TISEAN software (Hegger, Kantz \& Schreiber, 1999). The methods were applied to a Lorenz series, which resembles the dynamics found by Kelly, Heathcote, Heath and Longstaff (2001) in series of decision times. The Lorenz series was corrupted with up to $80 \%$ additive Gaussian noise, a lower signal-to-noise ratio than has been used in any previous test of these methods, but consistent with Kelly et al.'s data. Prediction methods performed the best for detecting nonstationarity and nonlinear dynamics, and optimal predictability provided an objective criterion for setting the parameters required by the analyses. Local linear filtering methods preformed best for characterization, producing informative plots that revealed the nature of the underlying dynamics. These results suggest that a methodology based on delay-space averaging and prediction could be useful with noisy empirical data series.

\section{Keywords: Nonlinear Dynamics, Fractal Dimension, Prediction, Time Series, Measurement Noise}


Penultimate Draft of a paper in press in Nonlinear Dynamics in Psychology and the Life Sciences, 2005

The study of mathematical chaos has provided useful new techniques for the analysis of time series (e.g., Hegger, Kantz \& Schreiber, 1999) and a framework for models of complex behavioural processes (e.g., Gregson, 1988, 1992, 1995; Guastello, 1995; Heath, 2000; Kelso, 1995; Newell, Liu \& Mayer-Kress, 2001). However, most of these techniques were developed for the physical sciences where the underlying determining equations are usually known and measurement noise can be minimised. In the behavioural sciences, in contrast, determining equations are rarely known, and it is difficult to eliminate measurement noise because measured systems cannot be sufficiently isolated to remove the effects of influential environmental variables.

This paper addresses the application of nonlinear dynamical analysis (NDA) techniques to behavioural data with particular reference to the problem of measurement noise. The usual response to noise in the behavioural sciences is averaging. However, when behaviour is nonlinear, inappropriate averaging can introduce distortion so that the average is not representative of any individual's behaviour (e.g., Brown \& Heathcote, 2003; Heathcote \& Brown, 2004; Heathcote, Brown \& Mewhort, 2000). Once the necessity of studying the individual is accepted, one is faced with a daunting level of variability in behaviour. Gilden (1997), for example, found that changes in the mean accounted for only $10 \%$ of individual variance in a range of choice response time (RT) experiments. Discarding the remaining $90 \%$ of variation as "error" assumes that no systematic explanation is possible for the vast majority of individual behaviour. Nonlinear dynamics, which can generate complex and apparently random behaviour while obeying relatively simple deterministic equations, provides an alternative conceptualisation of behavioural variability. As Luce (1995) states: “... the findings of 
Penultimate Draft of a paper in press in Nonlinear Dynamics in Psychology and the Life Sciences, 2005

the past 10 to 15 years about nonlinear dynamic systems call into question whether the actual source of the noise is randomness or ill-understood dynamics." (p.24).

However, it is unlikely that chaotic dyna mics can explain all of the variability of individual behaviour. The research reported here was inspired by studies of sequential dependences in the times to make series of simple choices, which under the right conditions can show evidence for nonlinear dynamics (Kelly, Heathcote, Heath \& Longstaff, 2001), but also clearly contained high levels of measurement noise. Where genuine noise is present, measurement of dynamics becomes problematic.

\section{Nonlinear Dynamics}

Figure.1a is an example of low-dimensional mathematical chaos, a time series from the Lorenz equations (see caption for details), which will be used to illustrate nonlinear dynamics throughout this paper. The Lorenz series was chosen because its dynamics resemble those observed by Kelly et al. (2001). The Lorenz series exhibits a pair of complex oscillatory states, with rapid transitions between these states occurring at apparently irregular intervals. Within each state, oscillations increase from small to large amplitudes before undergoing a state transition. The series in Fig.1a begins with a rapid state transition then spends a long time in the lower state, but later in the series the upper state has the majority of extended oscillations. The first major section of this paper reviews a range of NDA techniques and applies them to the Lorenz series. We then examine NDA for noisy series of the type that might be expected in behavioural data, by adding Gaussian noise to the series in Fig.1a.

Insert Figure 1 about here 
Penultimate Draft of a paper in press in Nonlinear Dynamics in Psychology and the Life Sciences, 2005

It should be emphasised from the outset that is difficult to provide a definitive step-by-step guide that covers NDA for all types of nonlinear dynamics. The very complexity that makes nonlinear dynamics attractive for modelling also means that caution is required in generalising findings about a particular type of chaotic series. Rather, the aim of this paper is to illustrate an investigative methodology that readers can adapt in order to ascertain if NDA will be useful with their own data, perhaps aided by analysis of model time series. The analysis software used here (with commands set in Courier) is available free through the TISEAN project (Version 2.1, http://www.mpipks-dresden.mpg.de/ tisean, see Hegger, Kantz \& Schreiber, 1999).

\section{Methods for Dynamical Analysis}

Chaos can range from low dimensional to high dimensional. Although noise and high dimensional chaos are conceptually distinct, they are almost impossible to distinguish in practice. Low dimensional chaos can be distinguished from noise, but obtaining data of sufficient quality can be difficult. The time series must be long, sampled at regular time intervals, and stationary (stationarity is defined below). Initially, NDA techniques were developed for physical science applications that yielded such series. In the life sciences, however, long stationary series are difficult to obtain, due to processes such as learning and fatigue, and high levels of measurement noise are often present. Genuine noise, particularly linearly autocorrelated noise, is problematic as it can appear like chaos to some NDA techniques.

The Fourier power spectrum of the series in Fig. 1a illustrates why noise is problematic for NDA. Fourier analysis is a traditional approach to such apparently struc tured oscillatory signals, allowing for dominant frequencies to be identified through peaks in the spectrum, and broadband noise to be removed through band-pass filtering. 
Penultimate Draft of a paper in press in Nonlinear Dynamics in Psychology and the Life Sciences, 2005

However chaotic signals can themselves be broadband, and so frequency domain filters are ineffective. Where noise is white (i.e., each sample is independent) the spectrum is flat. Coloured noises, such as are produced by linear and nonlinear autocorrelation produce spectra, are characterised by a decrease in power with increasing frequency. The Lorenz series also displays a decrease in power as frequency increases. The similarity of spectra for chaos and coloured noises makes them hard to distinguish.

Whereas Fourier decomposition transforms temporal information into frequency information, most NDA techniques rely on the transformation of temporal information into a geometrical representation: delay embedding. An $\underline{m}$ dimensional delay embedding converts a one dimensional time series, $x(t)$, to a set of $\underline{m}$ dimensional points $(x(\underline{t}+\delta)$, $\mathrm{x}(\underline{\mathrm{t}}+2 \delta), \ldots \mathrm{x}(\underline{\mathrm{t}}+\underline{\mathrm{m}} \delta))$, where $\delta$ is the time delay between samples. Takens' (1981) theorem states that a one to one image of the $\underline{\mathrm{d}}$ dimensional set of points visited by a stationary dynamical system (its attractor) can be reconstructed from an embedding in a delay space with dimension $\underline{\mathrm{m}}>2 \underline{\mathrm{d}}$. For the Lorenz system, for example, $\underline{\mathrm{d}} \approx 2.05$, with the attractor being a fractal subset of the space defined by the three Lorenz variables (i.e., $(\underline{x}, \underline{y}, \underline{z})$ of which only $\underline{x}$ is shown in Fig.1a). The complex, fractal nature of this set for chaotic systems have led to them being described as "strange attractors". Since Takens' initial result, "embedology" has been an active area of theoretical development, with Sauer, Yorke and Casdagli (1991) showing that more general schemes than simple delay coordinates can be used, including delay embeddings of series transformed by singular value decomposition and geometric filtering (Grassberger, Hegger, Kantz, Schaffrath \& Schreiber, 1993). 
Penultimate Draft of a paper in press in Nonlinear Dynamics in Psychology and the Life Sciences, 2005

Stationarity is essential for establishing an embedding. For a stochastic process, $y(t)$, stationarity can be defined as invariance of all finite-dimensional joint distribution functions of $\left(y\left(t_{1}\right), y\left(t_{2}\right), \ldots y\left(t_{m}\right)\right)$ over shifts on the temporal dimension (e.g., $\left.\left(t_{1+\Delta} \ldots t_{m+\Delta}\right)\right)$ (Rao \& Gabr, 1984). In the context of NDA, Casdagli (1997, p. 12) stated that for practical purposes: “...a time series $\underline{\mathrm{x}}_{1}, \underline{\mathrm{x}}_{2}, \ldots, \underline{\mathrm{x}}_{\mathrm{N}}$ is nonstationary if, for low $\underline{\mathrm{m}}$, there are variations in the estimated joint distribution of $\underline{\mathrm{x}}_{i}, \underline{\mathrm{x}}_{i+1}, \ldots, \underline{\mathrm{x}}_{i+\mathrm{m}-1}$ that occur on time scales of order N". An embedded representation can only translate all temporal information to spatial information if the underlying process is stationary. Given stationarity, NDA can recover aspects of the dynamics using geometrical analyses of the embedded set. The analyses often involve estimating the properties of local neighbourhoods or sets of points, with a neighbourhood defined on a distance measure in the embedding space. Where such analyses aggregate local measures to estimate global properties of the attractor they strongly rely on the assumption of stationarity. Aggregation is particularly important to counter the effects of measurement noise.

Even where stationarity holds, it should be acknowledged that a particular data series may not be suitable for NDA if all regions of the dynamics are not sampled, and hence the full joint distribution cannot be estimated. As in conventional statistics, one needs a sufficient number of replicates to form a reliable model, so intermittent chaos or noisy periodic behaviours cannot be characterized when few intermittent events or periods are sampled. In some circumstances, even arbitrarily small amounts of noise can destroy the embedding property (Casdagli, Eubank, Farmer \& Gibson, 1991). However, delay coordinates are also useful when an embedding is not required, such as in the prediction of nonlinear time series with both deterministic and stochastic dynamical 
Penultimate Draft of a paper in press in Nonlinear Dynamics in Psychology and the Life Sciences, 2005

structure (Weigend \& Gershenfeld, 1993). As will be discussed later, NDA based on prediction is especially attractive because it remains useful when noise levels are high.

Figure 2a illustrates the nonlinear dynamics of the Lorenz time series, by plotting each value against an estimate of its first derivative, obtained by successive ("lag one") differences. The derivative plot shows that the series change s quickly near the centre of each oscillation state, and more slowly in the region between states, and at the positive and negative extremes. The spikes to the left and right of the main body of the plot are produced by very large changes, a large decrease for the positive state and three large increases for the negative state. The derivative plot also shows that the initial 2000 or so observations in the series have different dynamics to the remainder of the series. The series first exhibits an unusually large decrease then oscillates for an extended period around the centre of the lower state. The values of the series from $t=2001-10000$ will be used to illustrate the analysis of stationary dynamics, with the first 2000 observations used to illustrate the detection of nonstationarity.

Insert Figure 2 about here

Most NDA techniques are based on time delay coordinates, so the y require the user to select a delay, embedding dimension, and often other parameters. Parameter selection is often difficult because the best choice depends both on the data set and the technique. A range of methods have been devised to select parameters for a given data set; however, the methods can also require parameter values to be selected. Further, both the dynamical analysis and parameter selection methods can fail when noise is present. In practice, NDA for empirical data is still as much an art as a science. 
Penultimate Draft of a paper in press in Nonlinear Dynamics in Psychology and the Life Sciences, 2005

The following sections survey a range of methods for performing linear and nonlinear dynamical analyses. The first section examines linear methods, which are a necessary preliminary to nonlinear analysis. The following section discusses measures of nonlinearity based on the ideas of recurrence and sensitive dependence. The final section discusses inferential testing for the presence of nonlinearity, and filtering methods that remove noise, enabling the graphical characterization of dynamical structure.

\section{Linear Dynamical Analysis}

Linear dynamical analyses can be accomplished using standard ARMA (AR=autoregressive, MA=moving average) modelling (Box \& Jenkins, 1976; Cryer, 1986). Linear autoregressive models create future values from linear combinations of past values and noise, producing linear autocorrelations between past and future observations. Most chaotic models have the same type of recursive dynamics, except that the combination also contains nonlinear components. As a result, chaos almost always produces strong linear autocorrelations. The best fitting linear autoregressive model for the series in Fig.1a, as determined by the number of significant partial autocorrelation coefficients, is of order 12 and accounts for more than $99 \%$ of the variation in the series. However, the residuals for this model are structured, as it does not account for all of the increasing magnitude of oscillations within a state, with four sets of large deviations correspond ing to the large differences in Fig.2a. Fits requiring large numbers of parameters (e.g., 12 compared to the 3 parameters of the Lorenz equations), and failure to account for fine-grained structure, are characteristic of ARMA models of chaotic series.

De-trending, through transformations such as taking successive differences or subtracting a regression estimate of the mean, is usually applied because ARMA analysis assumes second order or "weak" stationarity: constancy of the mean, variance and auto- 
Penultimate Draft of a paper in press in Nonlinear Dynamics in Psychology and the Life Sciences, 2005

covariance $^{1}$. The Lorenz series also displays nonstationarity up to around 2000

observations, evident in an increase in the local mean of the series (the thick line in

Figure 1a). However, despite a broad averaging window of 1000 observations, the local mean continues to fluctuate appreciably throughout the series. The se fluctuations do not reflect nonstationarity, but rather the underlying nonlinear dynamics, which spends varying amounts of time in the upper and lower states. Similar observations can be made about the local variance of the series, so in this case neither the local means nor variances are useful for detecting the initial nonstationarity.

For the Lorenz series, the initial nonstationarity did not make much difference to the fitted AR model, but the autocorrelation function was somewhat shorter when the first 2000 observations were omitted. The length of an autocorrelation function is often characterised by its first zero, or by the "correlation time", the lag at which it drops to 1/e $(\approx 0.378)$ of its initial value. Chaotic series can have very long autocorrelation functions. For the full series in Fig.1a autocorrelations up to lag of 500 did not cross zero and had a correlation time of 41 . With the first 2000 values omitted the autocorrelation function crossed zero at lag 314 and had a correlation time of 31.

De-trending must be applied cautiously as a preliminary to NDA. For example, subtracting the local mean averaged at the scale illustrated in Fig.1a would destroy information about the underlying dynamics. In particular, NDA should not be carried out on residuals from ARMA analysis (Theiler \& Eubank, 1993). Chaotic dynamics often produce linear components, and ARMA models with a large number of parameters can fit some variation due to nonlinear components, so that residuals do not contain the information necessary for NDA. However, NDA does rely on comparisons with linear 
Penultimate Draft of a paper in press in Nonlinear Dynamics in Psychology and the Life Sciences, 2005

models. Instead of examining residuals, an experimental series is compared to

"surrogate" series, formed by randomising the experimental series while maintaining structure dictated by a null hypothesis, such as linear structure (Theiler, Eubank, Longtin, Galdrikian \& Farmer, 1992), as described below.

\section{Nonlinear Methods based on Recurrence}

Graphical methods are particularly useful for revealing recurrent, or close to recurrent, structure in time series. A delay plot graphs a time series, $\mathrm{x}(\mathrm{t})$, against a delayed version of itself, $\mathrm{x}(\mathrm{t}+\delta)$, where $\delta$ is the delay. Periodic attractors display exact recurrence, returning to the same state or states at regular intervals. Hence, for appropriately chosen delay intervals, such systems can be represented by a single point or small set of points in a delay plot. Chaotic systems are not exactly recurrent because they contain many unstable periodic orbits, but delay plots can still reveal behaviour that is close to recurrent, and so they are useful for understanding the qualitative dynamics of a chaotic system.

Note that delay plots and derivative plots (e.g., Fig.2) contain the same information. For example, the lag-one derivative estimate for a point in a lag-one delay plot equals the difference between vertical and horizontal distances from the right diagonal. The derivative plot has the advantage of a direct interpretation in terms of process dynamics, and can be extended using estimates of higher order derivatives. However, estimates of higher order derivatives can be very variable when noise is present, so attention is restricted here to the first derivative.

Figure 2 shows that an appropriately chosen delay provides a clear representation of temporal structure in derivative plots. When the delay is short (e.g., Fig.2a) orbits are too closely packed to be discriminable, whereas when the delay is too long, details may 
Penultimate Draft of a paper in press in Nonlinear Dynamics in Psychology and the Life Sciences, 2005

be lost in some regions. In general, no single delay necessarily provides the best detail of all regions of the dynamics (cf. Hegger's et al., 1999, Fig.1). The embedding theorem should apply for any delay, but most NDA techniques require specification of an appropriate delay in order to avoid the redundancy evident in Fig.2a. Series of derivative or delay plots can be used to choose the delay producing the clearest structure. For the Lorenz data, a delay of 12 provided the best result, agreeing with the order of the AR model for this time series.

Methods of choosing delay based on autocorrelation, such as the correlation time or the first zero of the autocorrelation function, are sometimes recommended. However, these estimates, given earlier, were not useful for the Lorenz series. Time delayed mutual information (i.e., the information shared by lagged versions of a time series) is also useful for estimating delay (Fraser \& Swinney, 1986). Mutual information accounts for both linear and nonlinear structure, with the delay being set to its first minimum as a function of lag. For the stationary Lorenz series, the resulting estimate was 17 (mutual lorenz2. dat -D100 -o provides mutual information to 100 lags), somewhat longer than the linear estimate of 12 , reflecting the longer time scale of the nonlinear interactions. For the following analyses of the deterministic Lorenz series little difference was observed for delays ranging from 12 to 17.

Recurrence plots, which were originally proposed by Eckmann, OliffsonKamphorst and Ruelle (1987), provide an alternative means of graphical analysis. They are constructed by plotting points (on a grid defined by two time axes) where recurrence nearly occurs in an $\underline{\mathrm{m}}$ dimensional embedding. Recurrence was originally defined as being a $\underline{\mathrm{k}}$-th nearest neighbour, but a definition based on a minimum distance is more 
Penultimate Draft of a paper in press in Nonlinear Dynamics in Psychology and the Life Sciences, 2005

often used (Koebbe \& Mayer-Kress, 1991). The TISEAN command recurr produces an output file that can be used to create a recurrence plot of the latter type. Recurrence plots produce visual patterns that may be useful in detecting nonstationarity, but general guidelines for how to interpret the patterns are difficult to formulate (cf. Schreiber, $1999)^{2}$.

Dimensionality estimates provide quantitative indices of nonlinear dynamics based on topological regularities such as near recurrence. According to Takens' (1981) theorem, the minimum dimension guaranteed to establish an embedding is given by the smallest integer $\underline{\mathrm{m}}>2 \underline{\mathrm{d}}$, where $\underline{\mathrm{d}}$ is the possibly fractional dimension of the attractor that generated the time series. Simple periodic attractors have a dimension of one, and quasiperiodic attractors, such as tori, have higher integer dimensions. Chaotic dynamics have attractors with non-integer dimensions, because they fill the phase space in a fractal manner with an invariant distribution of points on the attractor at different length scales.

The Lorenz equations operate in a 3 dimensional space, but the Lorenz attractor has dimension, $\underline{\mathrm{d}} \approx 2.05$. A value of $\underline{\mathrm{m}}=5$ will be adopted here for the further analysis of the Lorenz data, in accord with Takens' theorem. Note, however, that Takens' theorem does not dictate that smaller values of $\underline{m}$ will not work. For the Lorenz system, for example, $\underline{m}=3$ can be sufficient to achieve an embedding. Heath (2000) favours the False Nearest Neighbour method (Kennel, Brown \& Abarbanel, 1992) to determine a minimum embedding dimension. Estimation of a minimum embedding dimension will be discussed once some background information has been established.

A number of different attractor dimensionality estimates are available, based on either the correlation sum or information theory measures. For example, dimensionality 
Penultimate Draft of a paper in press in Nonlinear Dynamics in Psychology and the Life Sciences, 2005

may be estimated using D1, the information dimension, and the dynamics of two series may be compared using Kullback entropy (see Schreiber, 1999). We will examine the most commonly used estimate of dimensionality, D2. D2 is based on the correlation sum, $\mathrm{C}(\varepsilon)$, which measures the proportion of embedded points that fall within a given distance $(\varepsilon)$ of each other (Grassberger \& Procaccia, 1983). The correlation sum measures recurrence at a given distance or scale, so it is equivalent to the proportion of points marked as recurrent at a given distance on a recurrence plot.

D2 summarises the way the correlation sum changes as a function of distance. It can be estimated from the exponent of a power law relationship between the correlation sum and distance. The exponent is usually measured by the slope of a $\log (\mathrm{C}(\varepsilon))-\log (\varepsilon)$ plot. Because the power law relationship never applies for all distances in a finite series (technically the dimension of a finite series is zero), the slope is assessed locally, that is, it is assessed for a restricted range of distances. For chaotic systems the slope increases with $\underline{\mathrm{m}}$, but then becomes constant once a proper embedding is achieved. The constant slope provides the D2 estimate. White noise, in contrast, consists of a series of independent random values, so it fills the delay space uniformly no matter what its dimension, and produces increasing slope estimates.

Early applications of NDA used finite D2 estimates as evidence for chaos. However, a finite D2 is a necessary but not sufficient condition for chaos. Coloured noises, which result from stochastic dependencies between series of values, also produce finite D2 estimates. Osborne and Provenzale (1989) showed that an embedded random walk has a dimension of two. Provenzale, Smith, Vio and Murante (1992) discuss these problems and provide examples of finite D2 estimates for both linear and nonlinear 
Penultimate Draft of a paper in press in Nonlinear Dynamics in Psychology and the Life Sciences, 2005

autoregressive models. Theiler and Rapp (1996) concluded that much of the supposed evidence for chaos relying on finite estimated dimensionality suffered from these problems. Hence, D2 estimates may be best used as a method of characterising rather than identifying nonlinear dynamics.

Even where nonlinear dynamics are present, measurement noise is particularly detrimental to D2 estimation, as it smears out the fine details of an attractor. As a result, accurate absolute dimensionality estimates are difficult to obtain. Relative measurements of dimensionality may, however, remain useful, as long as estimates are obtained with the same measurement function, $\mathbf{y}=f(\mathbf{x})$, where $\mathbf{y}$ is the observed time series, $\mathbf{x}$ is the series produced by the dynamics, and $f$ is a monotone function. Under ideal circumstances D2 is invariant with respect to the measurement function, but when only relative measurements are possible it may not be invariant. Hence, comparisons may be confounded if the measurement functions differ. Similarly, changes in the level and type of measurement noise may change D2 estimates, and so confound comparisons.

An important technical issue in D2 estimation, and other estimates based on the correlation sum, is that they assume that pairs of points are drawn randomly and independently according to the scale invariant measure of the attractor. Independence cannot apply for points occurring close in time, and if such points are included spuriously low estimates of D2 occur. To avoid the problem, points closer than some minimum time can be excluded from the correlation sum (Grassberger, 1987; Theiler, 1990). The number of points excluded, $\underline{w}$, is called the Theiler window. An easy estimate of the Theiler window can be obtained by multiplying the correlation time by three (Heath, 
Penultimate Draft of a paper in press in Nonlinear Dynamics in Psychology and the Life Sciences, 2005

2000). A more rigorous estimate is given by a space-time separation plot (Provenzale, et al., 1992).

A space-time separation plot is related to the correlation sum and the recurrence plot. It shows equal probability contours for the distribution of distances between pairs of points as a function of time. For a chaotic series the contours initially rise then oscillate around a constant value, whereas for coloured noise they continue to rise. Figure 3 shows a space-time separation plot for the Lorenz data, which displays contours of constant probability for the spatial separation of pairs of points in an embedding as a function of their separation in time. The Theiler window, $\underline{\mathrm{w}}$, is chosen at a time beyond which the constant behaviour is operating. For the following analyses $\underline{w}$ was set at a value of 100 , which approximately equals the estimate from three times the correlation time $(3 \times 31)$. Values in the range 50-300 were found to have a similar effect.

Insert Figure 3 about here

Figure 4 shows, for the stationary Lorenz series and a Theiler window of 100, local slope estimates of $\log (\mathrm{C}(\varepsilon)) \sim \log (\varepsilon)$ as a function of $\log (\varepsilon)$ for $1-10$ embedding dimensions. The slope in a "scaling region" (a range of distances with the same constant slope for all larger embedding dimensions) provides an estimate of dimensionality. The local slope plot is a useful alternative to directly estimating D2 for the slope of $\log (\mathrm{C}(\varepsilon)) \sim \log (\varepsilon)$ plots because it makes evident the extent of, and deviations from, scaling behaviour. Dimensionality cannot be estimated unless scaling behaviour occurs; the TISEAN programmers emphasise this point so strongly that they purposely do not provide any automatic estimate of dimensionality, only outputs for constructing plots. 
Penultimate Draft of a paper in press in Nonlinear Dynamics in Psychology and the Life Sciences, 2005

Insert Figure 4 about here

In Fig.4a the scaling region is broad, because estimates were based on 98000 stationary points. Scaling is achieved by an embedding dimension of three, and the slope in the scaling region agrees closely with the true dimensionality. As the computation of D2 scales as the square of series length it can be quite time consuming (e.g., the estimate for the 98000 length series took 3 hours on a 2.6GHz AMD Athlon XP computer). In Fig. $4 \mathrm{~b}$ the scaling region is much narrower, as the estimates are based on only 8000 stationary points, and was not achieved until an embedding dimension of 5. However, it is still clearly identifiable around a distance of 2 and only slightly overestimates the true dimensionality.

\section{Nonlinear Methods based on Sensitive Dependence}

An alternative approach to measures based on fractal topology comes from another defining characteristic of chaos: sensitive dependence on initial conditions. In chaotic systems initially similar points soon move far apart as trajectories diverge exponentially. Divergence occurs at a rate described by the Lyapunov spectrum. Globally stable chaotic systems (such as the Lorenz) are bounded overall, as the sum of their Lyapunov exponents is negative, but trajectories diverge in one or more dimensions at rates determined by the set of positive exponents. Methods are available to estimate the full Lyapunov spectrum (e.g., the Netle software, http://www.sfu.ca/ rgencay/lyap.html). However, Heath (2000) notes that these methods are not noise tolerant, and favours a more robust approach based on estimating only the maximum Lyapunov exponent, with a positive maximum exponent indicating chaos. We found that even this method fails in noisy series, so Lyapunov based methods are not further examined here. 
Penultimate Draft of a paper in press in Nonlinear Dynamics in Psychology and the Life Sciences, 2005

The False Nearest Neighbour method (Kennel et al., 1992), which is used to determine a minimum embedding dimension, is also based on the idea of examining the divergence of neighbouring points. For a range of embedding dimensions, the distance in the future (usually just one step) between each data point and its nearest neighbour is compared. If the ratio of the distance after one step to the original distance exceeds a criterion, the point is declared a false neighbour. The process is repeated for a range of embedding dimensions and the percentage of false neighbours plotted. For example, the TISEAN command false_nearest lorenz2.dat -M10 -d17 -o -t100 - 55 calculates the number of nearest neighbours with a ratio greater than 5 for $1-10$ dimensions. The result clearly indicates $\underline{m}=3$ is sufficient, a result that holds for a wide range of criterion ratios. A Theiler window of 100 was specified, as the false nearest neighbour technique makes similar assumptions to the dimensionality estimation techniques. A minimum embedding dimension can also be estimated from the D2 plots by the minimum number of dimensions necessary to attain a scaling region.

Sugihara and May (1990) suggested that predictability could be used to measure nonlinear dynamics in a time series. Although deterministic chaotic series are predictable in the short term, small initial differences due to measurement error are rapidly magnified by sensitive dependence, so that the series cannot be predicted in the long term. White noise, in contrast, is not predictable on any time scale. Sugihara and May suggested that decreasing predictability with time provides evidence for chaos. However, as with other NDA measures, stochastic temporal dependencies (e.g., coloured spectra produced by linear and nonlinear AR models) can confound results as they also cause a gradual decrease in predictability. 
Penultimate Draft of a paper in press in Nonlinear Dynamics in Psychology and the Life Sciences, 2005

To achieve prediction without knowledge of the underlying determining equations, Sugihara and May (1990) used a principle suggested by Lorenz (1969): similar present states in a deterministic system should evolve into similar future states. To compensate for the effects of noise, the evolution of a point in an embedding is predicted by the aggregate evolution of a set of neighbouring points, rather than just the evolution of the single most similar point (Lorenz's "analogue" point). Aggregation can range from simple averaging to more sophisticated combinations, and the size of the neighbourhood varied to match the level of noise. TISEAN provides the predict and zeroth programs, which use local averages, and onestep and nstep, which use local linear prediction. Barahona and Poon (1996) suggested a global polynomial prediction method for detecting chaos in short, noisy, time series. However, Schreiber and Schmitz (1997) report that when noise is high, local average techniques are better for this purpose.

\section{Surrogate Testing, Time Asymmetry and Geometric Filtering}

Estimators for most quantitative indices assume nonlinear dynamics are present, and can produce misleading results when they are not. Hence, it is important to test for, rather than assume, nonlinear dynamics in a time series. However, most measures of nonlinear dynamics can vary with the static distribution of the data and are also sensitive to linear dynamics. A bootstrap (e.g., Davison \& Hinkley, 1997) solution to these problems is provided by surrogate series testing (Theiler et al., 1992). Surrogate series are random variations on an experimental series that are constrained to preserve structure assumed under a null hypothesis. Permutations of the order of the experimental series, for example, produce surrogates that realize the null hypothesis of no temporal structure and the same static distribution of values as the experimental series. 
Penultimate Draft of a paper in press in Nonlinear Dynamics in Psychology and the Life Sciences, 2005

A linear ARMA process provides a more interesting null hypothesis. For example, Theiler et al.'s (1992) Amplitude Adjusted Fourier Transform (AAFT) surrogates have as a null hypothesis a gaussian ARMA process observed through a static, possibly nonlinear, but invertible measurement function. Allowing for a monotonic nonlinear measurement function provides considerable flexibility to accommodate whatever static distribution characterizes the measured series. Schreiber and Schmitz (2000) developed an iteratively refined AAFT surrogate, computed by the TISEAN program surrogates, which is more accurate in matching both the spectrum and distribution of a finite data set.

A test is constructed by comparing the surrogate series to the experimental series on a measure sensitive to nonlinearity. Significance is determined by the percentile attained by the nonlinear measure for the experimental series in the distribution of estimates obtained from the surrogates. In a survey of measures of nonlinearity, Schreiber and Schmitz (1997) found that nonlinear prediction error, obtained using a local averaging technique such as the TISEAN predict algorithm, was the most consistent in discriminating a range of noisy chaotic times series (see also Tong, 1990, for a review of tests of linearity). For nonlinear prediction error, the test is one tailed, with prediction error for the experimental series at the $\mathrm{p}$ 'th percentile allowing rejection of the null hypothesis with confidence $100-\underline{p}$.

A two-tailed test can be made using a cubic time reversal index, which measures the asymmetry of the distribution of differences between series values at a fixed delay using a statistic related to the third cumulant around zero (e.g., the TISEAN timerev command calculates the sum of cubes of the differences divided by their sum of squares). 
Penultimate Draft of a paper in press in Nonlinear Dynamics in Psychology and the Life Sciences, 2005

This statistic is based on the theory of polyspectra (Rao \& Gabr, 1984), which generalizes gaussian linear ARMA and Fourier models to include moments of order greater than two. Stationary linear gaussian processes have a symmetric difference distribution and zero cumulants of order higher than two, so nonlinearity or nonstationarity is indicated by either a significantly positive or negative estimate relative to the surrogate distribution.

Schreiber and Schmitz (1997) found the cubic time reversal index to have low power with noisy Lorenz $\underline{x}$ series data, although it performed well for other chaotic series. The Lorenz series shown in Fig.1a yielded cubic time reversal indices of 0.396 and 0.381 for delays 1 and 12 respectively. At a delay of one, the difference distribution contains three very large positive values, which dominate the time reversal index because of the cubing operation (the index is 0.007 with these values removed). The presence of such large outliers exacerbates the low efficie ncy of higher order cumulants for estimating distribution properties (cf. Ratcliff, 1979), perhaps explaining the low power with the Lorenz $\underline{x}$ series found by Schreiber and Schmitz.

Diks, van Houwelingen, Takens and DeGoede (1995) discuss indices based on the reversibility of linear time series and suggest a potentially more efficient kernel based symmetry measure. Stam, Pinj and Pritchard (1998) suggested an alternative approach that combines prediction and time asymmetry without the need for surrogates. Prediction is compared for the original $(x(t), t=1 \ldots N)$ and a time reversed version of the original $(y(t)=x(N-t+1))$, with any difference being attributable to nonlinearity. They found that their time asymmetry technique produced strong discrimination with the chaotic Rossler equations, even with $50 \%$ additive Gaussian noise. No matter how efficient the statistic it 
Penultimate Draft of a paper in press in Nonlinear Dynamics in Psychology and the Life Sciences, 2005

is important to note that assymetry is only a sufficient indicator of nonlinearity, it is a not a necessary condition, so some nonlinear dynamics may display little asymmetry.

Although surrogate testing is extremely useful, results should be interpreted with caution. Chaos does imply nonlinearity as measured by appropriate surrogate tests, but nonlinearity does not necessarily imply chaos. Nonstationary linear processes and nonlinear stochastic processes may also cause a positive finding, as may non-monotonic measurement functions. For example, Schreiber and Schmitz (2000) showed that a stationary second order linear autoregressive process observed through a measurement function made noninvertible by taking successive differences, a common procedure in time series analyses, produces a positive surrogate test result. Noninvertible measurement functions may arise in other cases, such as the measurement of signal power (i.e., squared amplitude).

Spurious findings of nonlinearity may also occur if a time series is unevenly sampled or contains missing values (Schmitz, \& Schreiber, 1999). The surrogate generation method of Schreiber (1998) may be applied to these cases. This method can create surrogate series for any null hypothesis that can be expressed as a cost function (computed by the TISEAN program randomize). Although this method is quite general, it uses a simulated annealing algorithm, and so can be very computationally expensive. Stam, Pijn and Pritchard (1998) also show that AAFT surrogate testing may produce false positive results where strong periodicities are present. They demonstrate that this problem can be fixed if the series is truncated to a length that is an integer multiple of the dominant period. However, this also usually requires that a more computationally expensive Discrete Fourier Transform be used for surrogate generation, 
Penultimate Draft of a paper in press in Nonlinear Dynamics in Psychology and the Life Sciences, 2005

rather than the Fast Fourier Transform usually employed by AAFT algorithms.

Schreiber's (1998) cost function based surrogate algorithm can also address problems caused by periodic components, again at an increase in computational cost.

Even with the available prediction and surrogate testing methods, noise remains extremely problematic for the characterization of nonlinear dynamics. Derivative plots are particularly useful for characterizing dynamics, but noise makes them difficult to interpret, so some sort of noise filtering is required. Traditional approaches to noise removal, such as frequency domain filters, cannot be applied to chaotic signals because they are, like noise, broadband. Instead, geometric filters (Grassberger et al., 1993), which smooth local neighbourhoods of the attractor, can be used. In each neighbourhood the signal is reconstructed using the first few principal components of a linearization, usually achieved by singular value decomposition to ensure numerical stability. Because chaotic signals mainly project onto the dominant components, whereas noise is distributed evenly across all components, the reconstructed signal has an improved signal to noise ratio. Corrections are usually also made for the effects of curvature and sensitive dependence (Schreiber, 1999) and the filter may be iterated.

Like surrogate testing, local projective geometric filters should be used cautiously, and in particular the filter should not be iterated too many times (Mees \& Judd, 1993). When deterministic dynamic structure is present it may be distorted by overfiltering. For example, Mees and Judd found that a circular attractor contracted substantially after 20 iterations. Over-filtering can create apparent structure from noise because of the finite length of measured time series. Although in infinite samples noise is evenly distributed in phase space, inhomogeneities can occur in finite samples that are 
Penultimate Draft of a paper in press in Nonlinear Dynamics in Psychology and the Life Sciences, 2005

expanded by the filtering process. Hegger et al. (1999) provide an example where pure gaussian noise produced a structured delay plot after 10 iterations. They advise that little more than three iterations should be required if chaos is present. As was the case for prediction, larger noise levels can be dealt with by using larger neighbourhoods, and by retaining fewer principal components.

\section{Nonlinear Dynamical Analysis of Noisy Data}

In this section we apply techniques described in the last section to time series corrupted by noise. Three noisy versions of the Lorenz series in Fig. 1a were created by adding independent zero mean gaussian deviates with a standard deviation (SD) of 6.2 (i.e., the SD of the series in Fig.1a from $\mathrm{t}=2000-10000$ ), 8.7 and 12.4, creating stationary series with a signal to noise ratios of 1:1 (50\% noise, data file $\mathrm{n} 1$. dat), 1:2 (66.6\% noise, data file n2. dat ) and 1:4 (80\% noise, data file $n 4$.dat ). Linear analyses of the noisy series produced results consistent with the addition of white noise. The spectrum flattened, and the number of significant partial autocorrelation coefficients, and hence the order of estimated AR models, reduced as noise increased. No systematic patterning was evident in residuals from the AR models of the noisy series. Many of the NDA techniques discussed so far were not useful when applied to the noisy series; derivative

plots were unstructured at all delays, no clear first minimum could be found in the mutual information, and false nearest neighbour plots gave no clear indication of a minimum embedding dimension.

\section{Testing for Stationarity}

The first task, and perhaps the most difficult and crucial task in the dynamical analysis of behavioural data, is establishing stationarity. Stationarity is an important requirement for NDA, and when it does not hold, or cannot be shown to hold, one must 
Penultimate Draft of a paper in press in Nonlinear Dynamics in Psychology and the Life Sciences, 2005

be sceptical about the validity of results. Unfortunately, stationarity checks are inherently vulnerable to noise because they are based on the comparison of sub-sequences of a series, and so sample size is reduced. As illustrated by Fig.1a, weak stationarity checks (on the mean, variance, and auto-covariance) are not very discriminating of nonstationarity in the Lorenz series. What is required is a method of checking whether the full joint probability distribution, rather than just its lower order moments, is constant.

Schreiber's (1997) cross-prediction method of detecting nonstationarity is an attractive candidate, because neighbourhood aggregation can make prediction noise tolerant. This method determines the degree to which information about one segment of a time series allows prediction of another segment of a time series. If the time series is stationary and each segment sufficiently long, equal cross prediction should be possible, because each segment will be governed by the same joint probability distribution.

Figure 5a illustrates the mean squared error for one-step prediction for the first 10000 values of the noiseless Lorenz series broken into 10 consecutive segments of length 1000 . The algorithm used locally constant prediction in delay-17 five-dimensional coordinates, averaged over a minimum of 30 neighbours, to generate one-step-ahead predictions. Larger embedding dimensions and neighbourhood sizes produced a similar pattern of results, as did smaller delays. The cross prediction method correctly detects the initial nonstationarity as the series settles into the attractor and stationary behaviour thereafter. The result is most striking for the first 1000 values with the next 1000 showing only a slight effect. These results support the earlier decision to remove the first 2000 values from further analyses as a cautious response, although in practice the evidence might only support removing the first 1000 . 
Penultimate Draft of a paper in press in Nonlinear Dynamics in Psychology and the Life Sciences, 2005

Insert Figure 5 about here

Figure $5 \mathrm{~b}$ demonstrates that the cross prediction method still works when the series is corrupted with up to $80 \%$ noise. Prediction was quite poor overall because of high noise levels but the first segment is clearly singled out and even the second segment differs slightly. The parameters used were those that produced the best cross-prediction (i.e., prediction of other segments). Although this required some trial and error, the search was tractable as only two parameters were important: the embedding dimension and the neighbourhood size. For the $80 \%$ noise series, averages over a minimum neighbourhood size of 40 in 10 dimensional coordinates were best. In the $50 \%$ noise case, the predict default neighbourhood size of 30 was best at either 9 or 10 dimensions. For uniformity, 10 dimensions will be used for all noise levels. In all cases a delay of one was clearly superior to all other delays, indicating that longer delays are not required when noise is high. Further analyses of the noisy Lorenz series were carried out using the 8000 values from $\mathrm{t}=2001 \ldots 10000$.

These results demonstrate the utility of Schreiber's (1997) cross-prediction method with high noise levels and relatively short sub-sequences of 1000. In principle, more sophisticated prediction methods, or methods that are more appropriate given knowledge of the underlying dynamics, can be incorporated as they become available. The degree of averaging can be adjusted to suit the level of noise, and Schreiber claims that this approach can work with sub-sequences as short as 300-400. He presents crossprediction results in a similar way to recurrence plots, using grey scale to indicate the level of error, but in the present case the graphs in Fig.5 were clearer. 
Penultimate Draft of a paper in press in Nonlinear Dynamics in Psychology and the Life Sciences, 2005

An advantage of prediction methods is that parameters such as delay and embedding dimension can be chosen based on what gives the best predictions. Selection of parameters via minimising cross-prediction error is particularly appropriate as it avoids over- fitting (Browne, 2000), which can be an important problem because of the flexibility of local prediction techniques. Typically, it is wise to use a large embedding dimension with prediction methods so that all available information can be utilised. However, there is an increased computational cost associated with the higher dimensional representation. For the Lorenz data, the 10-dimensional delay-one coordinates that were optimal for cross-prediction were usually appropriate for the following analyses. Some adjustment was necessary, but in general this involved changing only a single parameter, making the process of finding appropriate settings tractable.

\section{Testing for Nonlinearity}

Surrogate tests aim to determine whether a series contains nonlinear structure, and so is a viable candidate for NDA. For each stationary noisy series, 99 surrogate series were created using Schreiber and Schmitz's (2000) iteratively refined AAFT algorithm (e.g., for $50 \%$ noise, surrogates n1. dat -n99 -o, which produces surrogates with names n1.dat_surr_\#, where \#=001..099). As discussed by Theiler, Linsay and Rubin (1993), a mismatch between the beginning and end of the series poses a problem for surrogate generation schemes that match linear autocorrelations in the original and surrogate series using Fourier methods. For finite series, Fourier amplitudes correspond exactly to the autocorrelation function only if the series is one period of a repeating sequence. Where this does not hold, tests of nonlinearity may produce false positives. Endpoint mismatch can be corrected by choosing a subsequence of the original 
Penultimate Draft of a paper in press in Nonlinear Dynamics in Psychology and the Life Sciences, 2005

series that matches end points as closely as possible (Ehlers, Havstad, Prichard \& Theiler, 1998).

TISEAN provides the program endtoend, which chooses a subsequence with a length that is a multiple of 2, 3 or 5 (as required by a Fast Fourier Transform), to minimises both mismatch and phase slippage between the beginning and end of a series. For the Lorenz data only the $80 \%$ noise series required truncation, as reported by the output of endtoend $\mathrm{n} 4$. dat, by removing the first 193 points, resulting in a series of length 7776 (n 4 s . dat), 2.8\% shorter than the original series. An alternative approach, useful where a matching subsequence cannot be found or the truncation required is too great, is to avoid Fourier based methods and directly match the second order autocorrelation structure using Schreiber's (1998) method.

Tests used the generally most discriminating measures of nonlinarity examined by Schreiber and Schmitz (1997): one-step prediction and cubic time assymetry. The multiple input file processing capability of the TISEAN predict and $t$ imerev programs was used to process the original and surrogate files in one pass ${ }^{3}$. Cubic time assymetry was not discrimative at any noise level and for differences at any delay, performing even worse than was reported by Schreiber and Schmitz for the Lorenz series. One possible reason is that Schreiber and Schmitz used noise created by phase randomisation so that the noisy series had the same spectrum as the original series. The gaussian noise used here has a different spectrum and produces some large deviates that result in increased sampling variability for the cubic time reversal index.

Locally constant prediction, in contrast, performed well, detecting the nonlinearity in the original sequence at a $97 \%$ level of confidence even for the $80 \%$ noise series, as 
Penultimate Draft of a paper in press in Nonlinear Dynamics in Psychology and the Life Sciences, 2005

shown in Fig.6. Figure 6 shows root mean square prediction error (RMSE) standardised using the mean and standard deviation of the surrogate distribution. $\mathrm{Z}$ scores for the original series were $-7.13,-3.18$ and -2.18 at $50 \%, 66,6 \%$ and $80 \%$ noise series. Some experimentation was required with the extent of neighbourhood averaging used by the TISEAN predict program. This program allows the radius of the neighbourhood to be specified either in absolute units or as a fraction of the series standard deviation. The later method (specified by the $-\mathrm{v}$ option) was used, as it automatically scales for the differing standard deviations of the noisy series.

Insert Figure 6 about here

To the nearest 0.1 , a neighbourhood size of 1.6 standard deviations produced the smallest RMSE for the original series, and for the 50\% (RMSE=6.879) and 66.6\% $(\mathrm{RMSE}=9.478)$ noise series. A delay-one 10-dimensional embedding was also optimal, in agreement with the cross-prediction findings, despite the larger neighbourhoods used here. The same setting also produced the best prediction for the $80 \%$ noise series $(\mathrm{RMSE}=13.058)$ but discrimination was poor, with the original series being placed only at the $18^{\text {th }}$ percentile. Larger neighbourhoods produced progressively better discrimination for the $80 \%$ noise series, with a radius of 2.6 producing the best discrimination, at the $3^{\text {rd }}$ percentile as shown in Fig.6c, but slightly poorer prediction (RMSE=13.316). For the other two series a broad range of neighbourhood sizes resulted in prediction error for the original series at the first percentile.

Stam et al.'s (1998) method, comparing nonlinear prediction for the original and time-reversed series, was also applied to the noisy Lorenz data. In all cases the timereversed data had a larger prediction error: $6.889,9.488$, and 13.038 for $50 \%, 66.6 \%$ and 
Penultimate Draft of a paper in press in Nonlinear Dynamics in Psychology and the Life Sciences, 2005

$80 \%$ noise with 1.6 SD neighbourhoods, and 13.322 for $80 \%$ noise with a 2.6 SD

neighbourhood. However, the differences were not nearly as large as reported by Stam et al. for the Rossler equations, or the average differences for the surrogate prediction tests. This method also suffers from the drawback that it does not provide a confidence level.

In an attempt to improve on the locally constant prediction used by the predict program, surrogate tests were also constructed using local linear model prediction error as calculated by the TISEAN onestep program. Most parameters were the same as for predict, but the best discrimination was produced with small minimum neighbourhood around 35 , where the original series is at the $4^{\text {th }}$ percentile and $Z=-2.03$. As with locally constant prediction, error decreased with neighbourhood size. The onestep program outputs prediction error divided by the series standard deviation, which decreased from 0.984 for neighbourhoods of 35 to 0.94 for neighbourhoods of 400 . As these results were barely on par with locally constant prediction, and their computation was at least an order of magnitude slower, predict seems preferable. Although not examined by Schreiber and Schmitz (1997), prediction error for more than one step was calculated to determine if better discrimination could be achieved. The results, shown in Fig.7, were costly to compute, requiring an overnight run for each series. They show that discrimination between the surrogates and the original series was substantially improved for longer prediction lags. Figure $7 \mathrm{~b}$ shows that, although a neighbourhood of 2.6 SD produced the best discrimination for lag one predictions, much better discrimination was produced at longer lags by a neighbourhood of 1.6 SD, which is also optimal for minimising overall prediction error. Figure $6 \mathrm{~d}$ shows the error distribution for lag 14 predictions, which produced the best discrimination 
Penultimate Draft of a paper in press in Nonlinear Dynamics in Psychology and the Life Sciences, 2005

across all lags, demonstrating the potential improvement in discrimination afforded by an examination of multiple prediction lags.

Insert Figure 7 about here

\section{Noise Filtering}

The analyses performed so far are important for the application of NDA to data with unknown properties because they validate two fundamental assumptions of most NDA techniques: stationarity and the presence of nonlinear dynamics. Passing both tests helps to ensure that the data are sufficient in terms of both quantity and quality for NDA and also validates the application of geometric filters to remove noise. The high noise levels in these examples defeated the direct application of quantitative indicators, such as dimensionality or Lyapunov coefficients, and qualitative indicators, such as derivative plots. However, with appropriate filtering, much of the structure in the original time series can be recovered (e.g., Fig.1b), derivative plots are structured (e.g., Figs.2c and 2d), and even D2 estimation reveals something close to a scaling region (e.g., Fig.4c), although it underestimates the Lorenz attractor's dimensionality. Before examining these results, however, it is useful to examine the behaviour of the filter itself.

Figure 8a shows the increase in the correlation between the original and filtered series as a function of the iteration of Grassberger et al.'s (1993) algorithm (e.g., ghkss n1. dat $-\mathrm{m} 10-\mathrm{d} 1-\mathrm{q} 2-\mathrm{k} 400-\mathrm{i} 9-0$, the $-\mathrm{i} 9$ and -0 parameters create files containing the filtered series for each iteration $n=1 \ldots 9$, with extensions . opt . $n$ ). The algorithm performs orthogonal projections onto a q-dimensional manifold in a neighbourhood of minimum size $\underline{\mathrm{k}}$ for each data point. Correlations are shown for three values of $\underline{q}, 1,2$ and 3, along with two minimum neighbourhood sizes, 400 and 800 , 
Penultimate Draft of a paper in press in Nonlinear Dynamics in Psychology and the Life Sciences, 2005

corresponding to neighbourhoods containing $5 \%$ and $10 \%$ of the series respectively. The delay-one 10-dimensional coordinates adopted in previous analyses also produced the highest correlations, but larger neighbourhoods were used for filtering than for crossprediction for two reasons. First, larger neighbourhoods can be used because the entire series of length 8000 is available, whereas only 1000 observations were available for cross-prediction. Second, larger neighbourhoods produced better correlations between the filtered and original series, although the improvement with increasing size reduced for larger neighbourhoods and less noise, so a difference between size 400 and 800 is only evident for the $80 \%$ noise series in Fig.8a.

Insert Figure 8 about here

Generally, increasing filter iterations and neighbourhood size, and lowering the dimensionality of the projections, produced smoother derivative plots (e.g., Figs.2c and 2d). Schreiber (1999) advises that even locally constant projections (-q0) may be used, but here derivative plots revealed some clear artefacts induced by filtering for locally constant and one-dimensional projections (-q1). The best results visually were produced by two-dimensional projections $(-q 2)$, which may be because this closely matches the dimensionality of the Lorenz series (2.05). However, the two-dimensional projection could cause some shrinkage of the underlying dynamics (cf. Mees \& Judd, 1993), as revealed by plots of the filtered series standard deviation in Figs.8b and 8c, and comparison of Figs.1a and 1b. Series filtered with three-dimensional projections, in contrast, almost exactly matched the standard deviation of the underlying dynamics at higher iterations, but not did correlate as highly with the original series as lower dimensional projections. These finding suggest that the asymptotic SDs of the filtered 
Penultimate Draft of a paper in press in Nonlinear Dynamics in Psychology and the Life Sciences, 2005

series for a range of projection dimensions may be used to determine what proportion of variance in a noisy series is due to nonlinear dynamics, and perhaps to provide a rough estimate of their dimensionality.

In applications, the information in Fig.8a is not available, but some guidance in parameter setting can be gained by examining SDs and derivative plots for the filtered series. Casdagli (1991) and Mpitsos (1994) have argued that the best evidence for low dimensional chaos is a complex but structured delay plot. As shown in Fig.2c, after six iterations using two-dimensional projections, the derivative plot of the filtered $50 \%$ noise series is quite structured, and contains smooth trajectories. The corresponding derivative plot using three dimensional projections (Fig.2d) is less clear and the trajectories less smooth, but important aspects of the dynamics are still evident, such as quicker changes near the centre of each state and slower changes between states and at extremes. Derivative plots for the higher noise series were correspondingly less structured, but appropriate filter setting still revealed the qualitative aspects of the dynamics. It is wise to always examine filtered series using derivative or delay plots, as some settings can produce artefacts, such as under filtering of the beginning and end of the series.

\section{Discussion}

The approach taken in this paper should be generally useful, but some of the specific conclusions are no doubt limited to the Lorenz dynamics and additive Gaussian noise. In practice, it is likely that an iterative approach will be required to refine theory and measurement techniques. As well as different dynamics, different noise models may be required, such as coloured noise or noise in dynamical parameters. Parameter noise can be particularly problematic as nonlinear systems can be extremely sensitive to some changes in parameters but virtually invariant with other parameter changes. Casdagli 
Penultimate Draft of a paper in press in Nonlinear Dynamics in Psychology and the Life Sciences, 2005

(1997) extended the idea of embedding to parameterised families of dynamical systems, allowing nonstationarity due to slowly varying parameters, and developed methods based on recurrence plots for reconstructing slowly varying parameter changes. Hegger, Kantz, Matassini, and Schreiber (2000) advocate "over-embedding” of such systems with $p$ varying parameters in an $\underline{\mathrm{m}}>2(\underline{\mathrm{d}}+\underline{\mathrm{p}})$ dimensional space.

An interesting possibility suggested by the results on stationarity testing is that separate blocks of trials in behavioural experiments or segments of physiological recordings can be concatenated in order to obtain a series of sufficient length for NDA. Often, avoidance of nonstationarity due to fatigue requires that measurement be broken up into blocks with rest periods between them. Longstaff and Heath (1999) report cases where separate segments of stationary chaotic time series can be concatenated without confounding NDA. In practice, it will not be known if each segment is a sample from the same stationary series, so a method of checking is needed. Conversely, it is often useful to detect change points, as they may signal underlying changes or the need for intervention. Cross-prediction stationarity checks can help to perform both functions. Although concatenation is attractive, and possibly necessary if NDA is to have impact in many areas of the life sciences, a number of questions spring to mind. Should embedding points that span segments be eliminated? Should segments be trimmed before concatenation to enforce constraints such as periodic continuity? To what degree does concatenation distort low frequency structure as its period approaches the length of subsequences? Hopefully future research will help to answer these questions.

The results of testing for nonlinearity were encouraging, although quite a deal of parameter tuning was required to produce sufficiently powerful tests. One relatively 
Penultimate Draft of a paper in press in Nonlinear Dynamics in Psychology and the Life Sciences, 2005

unexplored possibility opened up by these results is that higher power may be available at longer prediction lags. Although the results given in Fig.6d are promising, a post hoc approach like choosing the lag with the greatest discrimination will inflate Type 1 error. Should the use of longer prediction lags prove generally useful, a test that integrates over lags will be required, along with simultaneous confidence intervals, rather than the pointwise intervals shown in Fig.7.

For all prediction methods applied to noisy series a delay of one was found to produce the best results, contrasting sharply with the noiseless case where longer delays were preferred. One possible implication is that dynamics will be difficult to identify in noisy series unless they are finely sampled, as in Fig. 1. Coarse sampling may occur in practice if the dynamics evolve on a much faster time scale that the measured behaviour. A preliminary exploration of this issue, using a series of length 10000 created by sampling every $100^{\text {th }}$ value from the first million values of the Lorenz $\underline{x}$ series, found that the prediction based surrogate test was still able to detect nonlinearity even with $80 \%$ noise. As shown in Fig.7c, significant indications of nonlinear dynamics were obtained at only the first two lags, contrasting with the results for a finer sampling. These results are encouraging for applications where the coarseness of sampling is not within experimental control.

Although geometric filtering was very effective in removing noise and recovering the underlying nonlinear dynamics, clearly there is a good deal of art in producing "clean" series and derivative plots, with an attendant risk of inflated Type 1 error. The neighbourhood size and projection dimension of geometrical filters act in much the same way as kernel bandwidth and regression order do to control smoothness in nonparametric 
Penultimate Draft of a paper in press in Nonlinear Dynamics in Psychology and the Life Sciences, 2005

regression functions (e.g., Wand \& Jones, 1995). The two techniques are conceptually related, except that nonparametric regression operates in the time domain for a time series, whereas geometric filtering acts in the embedding space. The job of the analyst will be made much easier if automatic neighbourhood size selection methods can be developed, in much the same way that automatic bandwidth selection methods are now available for nonparametric regression. Fortunately, both global and local approaches to geometric filtering are very active areas of research (e.g., Roweis, \& Lawrence, 2000; Tenenbaum, de Silva \& Langford, 2000).

The filtering results reported here are encouraging because they enable graphical identification of qualitative features of the dynamics underlying a noisy series. However, filtering can both fail to remove sufficient noise and introduce systematic distortions that affect estimates of quantitative dynamical indices, such as D2. Figure 4c shows the best local slope plot that was found for the filtered $50 \%$ noise Lorenz series. Something approaching a scaling region occurred around a distance of 5-6, but the slope estimates did not converge and overestimate the Lorenz attractor's dimension. Another apparent scaling region occurred around a distance of 8-9 but it is very narrow and substantially underestimates the dimensionality of the Lorenz attractor. A possible reason for these problems is the use of a two-dimensional projection, which results in shrinkage of the underlying dynamics. Figure $4 d$ shows the results when filtering used three-dimensional projections (i.e., the series shown in Fig.2d). No proper scaling region is revealed, although the region around 10 is close and underestimates dimensionality only slightly.

If the present results are any guide, absolute estimates of D2 from filtered series are not likely to be very accurate, although they may suffice for relative comparisons. 
Penultimate Draft of a paper in press in Nonlinear Dynamics in Psychology and the Life Sciences, 2005

Caution should be exercised in the estimation of quantitative indices from filtered series, because, as with any smoothing technique, filtering can introduce systematic bias. Either bias corrections must be developed, or alternative noise tolerant indices used that can be applied to unfiltered series. One graphical technique associated with D2 estimation, the space-time plot was informative without filtering (e.g., Fig.3b). The same Theiler window was indicated as for the original series, and evidence consistent with deterministic dynamics provided: constant rather than increasing contours for longer time separations. Note, however, that increasing contours do not necessarily rule out deterministic dynamics, as they may be caused by a combination of deterministic and stochastic dynamics. In general, none of the techniques reviewed here can definitively differentiate nonlinear stochastic and chaotic processes. Cencini, Falcioni, Kantz, Olbrich and Vulpiani (2000) discuss this issue and a possible analytic approach.

In summary, the results presented here suggest that methods based on delay-space averaging and prediction provide the most powerful means to address problems caused by measurement noise. Although it will probably never be possible to prove chaos in a noisy measured time series, robust and powerful algorithms are now available to test for and quantify nonlinear structures rather than simply assuming it. Prediction based methods are attractive not only for their noise-tolerance, but also if the aim of a dynamic theory is to explain all systematic temporal variation, as they provide direct measures of the available structure that are relatively theory free, in the sense of not requiring knowledge of the form or parameters of determining equations. Geometrical filtering methods, which are also based on delay-space averaging, appear to provide the best means of graphically characterizing nonlinear dynamics in noisy time series. 
Penultimate Draft of a paper in press in Nonlinear Dynamics in Psychology and the Life Sciences, 2005

\section{ACKNOWLEDGEMENTS}

Initial work on this project was completed by the first author while writing a review of Heath (2000) (Heathcote, 2002) and supervising Dr. Alice Kelly's PhD project. We would like to thank the School of Behavioural Sciences, University of Newcastle, and an Australian Research Council Large Grant for funding support and the TISEAN project (Hegger, Kantz \& Schreiber, 1999) for making their software freely available. 
Penultimate Draft of a paper in press in Nonlinear Dynamics in Psychology and the Life Sciences, 2005

\section{Footnotes}

${ }^{1}$ Stationarity up to order $\underline{\mathrm{k}}$ dictates invariance only of joint moments up to order $\underline{\mathrm{k}}$ (Rao \& Gabr, 1984). For a linear gaussian process, $\underline{k}=2$ stationarity implies “strong” stationarity, invariance of the full joint probability distribution, as moments greater than two are zero. ${ }^{2}$ Recurrence quantification analysis (RQA, Trulla, Giuliani, Zbilut \& Webber, 1996; Webber \& Zbilut, 1994) provides a number of indices to quantify recurrence plots. Thomasson, Hoeppner, Webber and Zbilut (2001) claimed that RQA “does not require assumptions about stationarity, length or noise" (, p.94). However, the RQA indices are formed by aggregating local measures across a time series and aggregation does require stationarity if a rigorous meaning is to be attached to the aggregate values. Both series length and noise are important in obtaining precise estimates, and an embedding may not even be possible due to noise, so noise and length are relevant to RQA.

It is also difficult to decide what distance should be chosen to define recurrence, a critical parameter that has a very strong effect on the values of the RQA indices.

${ }^{3}$ timerev n1.dat n1.dat_surr_0?? - d1 > n1tr.dat and predict n1.dat n1.dat_surr_0?? -m10 -d1-v1.6 -o > n1pred.dat. The "??" characters are DOS single character wildcards, - $d$ specifies the delay, and the redirection of standard output (>) stores the measure of nonlinearity in a file. For the predict command, the $-m$ option specifies the embedding dimension, and - 0 writes predicted values to a separate output file with _pred appended to the name of each input file. 
Penultimate Draft of a paper in press in Nonlinear Dynamics in Psychology and the Life Sciences, 2005

\section{REFERENCES}

Barahona, M. \& Poon, C.-S. (1996). Detection of nonlinear dynamics in short, noisy time series, Nature, $\underline{381}, 215-217$.

Box, G. E. P. \& Jenkins, G. M. (1976). Time series analysis: Forecasting and Control. San Francisco: Holden Day.

Brown, S. \& Heathcote, A. (2003). Averaging learning curves across and within participants. Behaviour Research Methods, Instruments \& Computers, $\underline{35}$, 11-21.

Browne, M. W. (2000). Cross-validation methods, Journal of Mathematical Psychology, 44, 108-132.

Cleveland, W. S. (1979). Robust locally weighted regression and smoothing scatterplots. Journal of the American Statistical Association, 74, 829-836.

Cryer, J. D. (1986). Time Series Analysis, Boston: PWS-Kent.

Casdagli, M. (1991). Chaos and deterministic versus stochastic non-linear modelling. Journal of the Royal Statistical Society, Series B, 54, 303-328.

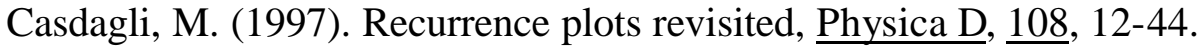

Casdagli, M., Eubank, S., Farmer, J. D. and Gibson, J. (1991). State space reconstruction in the presence of noise. Physica D, $\underline{51}, 52-98$.

Cencini, M., Falcioni, M., Kantz, M., Olbrich, E. \& Vulpiani, A. (2000). Chaos or Noise - Difficulties of a Distinction, Physics Review E $\underline{62}$, 427-437.

Cleveland, W. S. (1979). Robust locally weighted regression and smoothing scatterplots. Journal of the American Statistical Association, 74, 829-836.

Cryer, J. D. (1986). Time Series Analysis, Boston: PWS-Kent. 
Penultimate Draft of a paper in press in Nonlinear Dynamics in Psychology and the Life Sciences, 2005

Davison, A.C. \& Hinkley, D.V. (1997) Bootstrap methods and their application. Cambridge: Cambridge University Press.

Diks, C., van Houwelingen, J. C., Takens, F. \& DeGoede, J. (1995). Reversibility as a criterion for discriminating time series, Physics Letters A, 201, 221-228.

Eckmann, J. P., Oliffson-Kamphorst, S. \& Ruelle, D. (1987). Recurrence plots of dynamical systems. Europhysics Letters, $\underline{4}, 973$

Ehlers, C. L. Havstad, J. Prichard, D. \& Theiler, J. (1998). Low doses of ethanol reduce evidence for nonlinear structure in brain activity, Journal of Neuroscience, $\underline{18}$, 7474- 7486.

Fraser, A. M. \& Swinney, H. L. (1986). Independent coordinates for strange attractors from mutual information, $\underline{\text { Physical Review A, }} \underline{33}$, 1134-1140.

Gilden, D.L. (1997). Fluc tuations in the time required for elementary decisions. Psychological Science, 8, 296-301.

Grassberger, P. (1987). Evidence for climatic attractors: Grassberger replies, $\underline{\text { Nature, }} \underline{326}, 524$.

Grassberger, P. \& Procaccia, I. (1983). Characterization of strange attractors. Physical Review Letters, 50, 189-208.

Grassberger, P., Hegger, R., Kantz, H., Schaffrath, C. \& Schreiber, T. (1993). On noise reduction methods for chaotic data. Chaos, 3 , 127-141.

Gregson, R. A. M. (1988). Nonlinear psychophysical dynamics. Hillsdale NJ: Lawrence Erlbaum Associates.

Gregson, R. A. M. (1992). n-dimensional nonlinear psychophysics. Hillsdale NJ: Lawrence Erlbaum Associates. 
Penultimate Draft of a paper in press in Nonlinear Dynamics in Psychology and the Life Sciences, 2005

Gregson, R. A. M. (1995). Cascades and fields in perceptual psychophysics.

Singapore: World Scientific.

Guastello, S. J. (1995). Chaos, catastrophe, and human affairs: Applications of

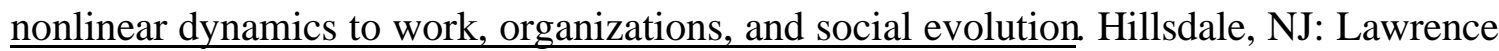
Erlbaum Associates.

Heath, R. A. (2000). Nonlinear Dynamics: Techniques and Applications in Psychology, Earlbaum: Mahwah, NJ.

Heathcote, A. (2002). Book Review, An Introduction to the Art; Nonlinear Dynamics: Techniques and Applications in Psychology by R. A. Heath, Journal of Mathematical Psychology, 46, 609-628.

Heathcote, A., \& Brown, S. (2004). Beyond curve fitting? Comment on Liu, Mayer-Kress and Newell (2003), Journal of Motor Behavior, 36, 225-232.

Heathcote, A., Brown, S. \& Mewhort, D.J.K. (2000) Repealing the power law: The case for an exponential law of practice. Psychonomic Bulletin and Review, $\underline{7}, 185-$ 207.

Hegger, R., Kantz, H., Matassini, L. \& Schreiber, T. (2000). Coping with

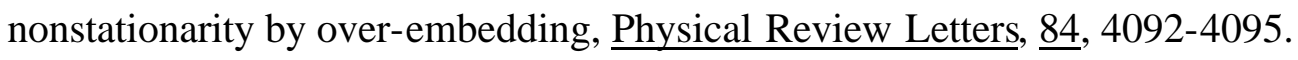

Hegger, R., Kantz, H. \& Schreiber, T. (1999). Practical implementation of nonlinear time series methods: The TISEAN package, $\underline{\text { Chaos, }}$ 9, 413-435.

Kelly, A., Heathcote, A., Heath, R. A. \& Longstaff, M. (2001). Response time dynamics: Evidence for linear and low-dimensional nonlinear structure in human choice sequences. Quarterly Journal of Experimental Psychology, 54, 805-840. 
Penultimate Draft of a paper in press in Nonlinear Dynamics in Psychology and the Life Sciences, 2005

Kelso, J. A. S. (1995). Dynamic patterns: The self-organization of brain and behaviour, Cambridge, MA: MIT Press.

Kennel, M. B. Brown, R. \& Abarbanel, H. D. I. (1992). Determining embedding dimension for phase-space reconstruction using a geometrical construction, $\underline{\text { Physical }}$ $\underline{\text { Review A, }} \underline{45,3403-3411 .}$

Koebbe, M. \& Meyer-Kress, G. (1991). Use of recurrence plots in the analysis of time-series data, M. Casdagli \& S. Eubank (Eds.) Nonlinear Modelling and Forecasting, Reading, MA: Addison-Wesley, 361-376.

Longstaff, M. \& Heath, R. A. (1999). A nonlinear analysis of the temporal characteristics of handwriting. Human Movement Science, $\underline{18}$, 485-524.

Lorenz, E. N. (1969). Atmospheric predictability as revealed by naturally occurring analogues. Journal of Atmospheric Science, 26, 636

Luce, R.D. (1995). Four tensions concerning mathematical modelling in psychology. Annual Review of Psychology, 46, 1-26.

Mees, A.I. \& Judd, K. (1993). Dangers of geometric filtering. Physica D, $\underline{68}$, 427-436.

Mpitsos, G.J. (1994). The chaos user's tool kit. Integrative Physiological and Behavioural Science, 29 , 307-310.

Newell, K. M., Liu, Y-T., \& Mayer-Kress, G. (2001). Time scales in motor learning and development, Psychological Review, 108, 57-82.

Nusse, H. E. \& Yorke, J. A. (1997). Dynamics: Numerical Explorations, $2^{\text {nd }}$ Edition, New York: Springer-Verlag. 
Penultimate Draft of a paper in press in Nonlinear Dynamics in Psychology and the Life Sciences, 2005

Osborne, A. R., \& Provenzale, A. (1989). Finite correlation dimension for stochastic systems with power-law spectra, Physica D, $\underline{35}, 357-381$.

Provenzale, A., Smith, L. A., Vio, R. \& Murante, G. (1992). Distinguishing between low-dimensional dynamics and randomness in measured time series, Physica D, $\underline{58}, 31-49$.

Rao, T. S., \& Gabr, M. M. (1984). An introduction to bispectral analysis and bilinear time series models, New York, Springer-Verlag.

Ratcliff, R. (1979). Group reaction time distributions and an analysis of distribution statistics. Psychological Bulletin, $\underline{86}, 446-461$.

Roweis, S. T. \& Lawrence, K. S. (2000) Nonlinear Dimensionality Reduction by Locally Linear Embedding, Science, 290, 2323-2326.

Sauer, T., Yorke, J. \& Casdagli, M. (1991). Embedology. Journal of Statistical $\underline{\text { Physics, }}$ 65, 579-616.

Schmitz, A. \& Schreiber, T. (1999). Testing for nonlinearity in unevenly sampled time series, Physical Review E, 59, 4044-4047.

Schreiber, T. (1997). Detecting and analysing nonstationarity in a time series using nonlinear cross predictions, $\underline{\text { Physical Review Letters, }}$ 78, 843-846.

Schreiber, T. (1998). Constrained randomisation of time series data, Physical $\underline{\text { Review Letters, }}$ 80, 2105-2108.

Schreiber, T. (1999). Interdisciplinary application of nonlinear time series

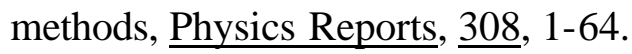

Schreiber, T. \& Schmitz, A. (1997). Discrimination power of measures for nonlinearity in a time series, Physical Review E, 55, 5443-5447. 
Penultimate Draft of a paper in press in Nonlinear Dynamics in Psychology and the Life Sciences, 2005

Schreiber, T. \& Schmitz, A. (2000). Surrogate time series, Physica D, 142, 346382.

Stam, C. J., Pijn, J. P. M. \& Pritchard, W. S. (1998). Reliable detection of nonlinearity in experimental time series with strong periodic components. Physica D, $\underline{112}, 361-380$.

Sugihara, G. \& May, R. (1990). Nonlinear forecasting as a way of distinguishing chaos from measurement error in time series, Nature, $\underline{344}, 734-741$.

Takens, F. (1981). Detecting strange attractors in turbulence: Lecture Notes in $\underline{\text { Mathematics, }} \underline{898}$, New York: Springer.

Tenenbaum, J. B., de Silva, V. \& Langford, J. C. (2000). A global geometric framework for nonlinear dimensionality reduction, Science, 290, 2319-2323.

Theiler, J. (1990). Estimating fractal dimension. Journal of the Optical Society of America A, $\underline{7}, 1055-1073$.

Theiler, J. \& Eubank, S. (1993). Don't bleach chaotic data. Chaos, $\underline{3}$, 335-341.

Theiler, J., Eubank, S., Longtin, A., Galdrikian, B. \& Farmer, J.D. (1992). Testing for nonlinearity in time series: The method of surrogate data. Physica D, 58, 77-94.

Theiler, J. Linsay, P. S. \& Rubin, D. M. (1993) Detecting nonlinearity in data with long coherence times, in A. S. Weigend and N. A. Gershenfeld, Eds., Time series prediction: Forecasting the future and understanding the past. Reading, MA: AddisonWesley.

Theiler, J. \& Rapp, P. E. (1996). Re-examination of the evidence for lowdimensional, nonlinear structure in the human electroencephalogram. Electroencephalographic Clinical Neurophysiology, 98, 213-222. 
Penultimate Draft of a paper in press in Nonlinear Dynamics in Psychology and the Life Sciences, 2005

Thomasson, N., Hoeppner, T. J., Webber, C. L. \& Zbilut, J. P. (2001). Recurrence quantification in epileptic EEGs, Physics Letters A, 279, 94-101.

Tong, H. (1990). Non-linear time series, a dynamical system perspective. Oxford: Oxford University Press.

Trulla, L. L., Giuliani, A., Zbilut, J. P. \& Webber, C. L. (1996). Recurrence quantification analysis of the logistic equation with transients, Physical Letters A, 223, 255-260.

Wand, M. P. \& Jones, M. C. (1995). Kernel Smoothing. London: Chapman \& Hall.

Webber, C. L. \& Zbilut, J. P. (1994). Dynamical assessment of physiological systems and states using recurrence plot strategies. Journal of Applied Physiology, $\underline{76}$, 965-973.

Weigend, A. S. \& Gershenfeld, N. A. (1993). Time series prediction: Forecasting the future and understanding the past. Reading, MA: Addison-Wesley. 
Penultimate Draft of a paper in press in Nonlinear Dynamics in Psychology and the Life Sciences, 2005

\section{FIGURE CAPTIONS}

Figure 1. a) $10000 \underline{x}$ values (thin line) for the Lorenz differential equations:

$d x / d t=10(y-x), d y / d t=x(28-z-y)$, and $d z / d t=x y-z 8 / 3$, solved with step

size $=0.01$ and starting from $(\underline{x}, \underline{y}, \underline{z}, \underline{t})=(0,1,0,0)$, with a Lowess $($ Cleveland, 1979) smooth using a 1000 element moving window (thick line). The Lorenz series was generated using the default settings of the Smalldyn program (http://keck2.umd.edu/dynamics, see Nusse \& Yorke, 1997). Analyses reported here examine the first 10000 values (data file lorenz1.dat), values from $t=2001-10000$ (lorenz 2 . dat) or values from $t=2001$ 100000 (lorenz 3.dat). (b) Filtered noisy Lorenz series from t=2001-10 000. Noise was additive Gaussian with the same standard deviation as the Lorenz series (i.e. $50 \%$ noise, $n 1$.dat). The geometric filter was iterated six times, retaining two singular values and using a minimum neighbourhood size of 400 in a 10 dimensions delay 1 embedding (ghkss n1.dat -i6 -q2 -k400-m10 -d1 -o n1q2k400.dat).

Figure 2. A plot of $x(t)$ from Figure 1a against its derivative estimated at (a) lag one, and (b) lag 12. Points in the derivative plot are joined to emphasise the trajectory of the dynamics. In (a) $t=1 \ldots 10000$, whereas for (b) and (c) $t=2001 \ldots 10000$. In (a) points for $\mathrm{t}=1 \ldots 2000$ are joined by a dotted line, and the four "spikes", one pointing the left from the top lobe and three pointing to the right from the bottom lobe, are caused by single points with unusually large differences. Lag 12 difference plots for (c) the filtered 50\% noise series shown in Figure $1 \mathrm{~b}$, and (d) the $50 \%$ noise series filtered with the same parameters as (c), but using a three rather than two-dimensional projection. 
Penultimate Draft of a paper in press in Nonlinear Dynamics in Psychology and the Life Sciences, 2005

Figure 3. Space-time separation plots showing contours of constant probability for spatial separation as a function of time separation from 1-500 for pairs of embedded point s from (a) the series in Fig.1a from $\mathrm{t}=2001-10000$ using a 5 dimensional delay 17 embedding

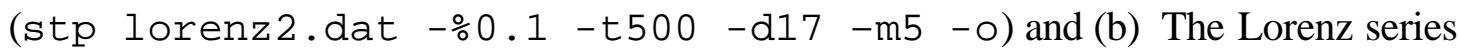
from $t=2001-10000$ with additive Gaussian noise of equal standard deviation and a 10 dimensional delay 1 embedding (stp $\mathrm{n} 1$. dat $-\mathrm{d} 1-\mathrm{m} 10$ - 0.1 -t500 - o). For parameter -\%f, the algorithm calculates $\mathrm{k}$ contours, where $\mathrm{k}$ is the nearest integer less than $1 / \mathrm{f}$, at probability values $1 / \mathrm{k}, 2 / \mathrm{k} \ldots 1$. A weakness of this algorithm is that it produces a noisy upper contour, as it is the sample maximum, which should not be given much weight in examining the pattern in the space-time plot. The contour for the maximum value has been omitted from (b) to provide clearer resolution of the lower contours.

Figure 4. Local slopes of $\log (\mathrm{C}(\varepsilon))$ vs. $\log (\varepsilon)$ plots for a range of distances, $\varepsilon$, and embedding dimensions from 1 to 10 in a delay 17 embedding with a Theiler window of 100 (a) for the Lorenz series for $t=2001-100000(d 2$ lorenz 3 . dat $-\mathrm{M} 1,10$ -d17 -t100 -N0 -o), (b) the Lorenz series for $t=2001-10000(\mathrm{~d} 2$ lorenz2. dat $-\mathrm{M} 1,10-\mathrm{d} 17-\mathrm{t} 100-\mathrm{N} 0-\mathrm{o})$, and the filtered noisy Lorenz series (c) using 2 dimensional projection, as in Figures $1 \mathrm{~b}$ and $2 \mathrm{c}(\mathrm{d} 2$ n1q2k400i6.dat -M1,10 -d17 -t100 -N0 -o) and (d) 3 dimensional projections, as in Figure 2d (d2 n1q3k400i6.dat -M1,10 -d17 -t100 -N0 - $\mathrm{O}$. The $-\mathrm{N} 0$ option indicated that the computation should be done on all possible pairs of points (the default is a maximum of 1000). Lower lines indicate lower embedding dimensions. The dashed line in each panel indicates the true dimension, 2.05 . 
Penultimate Draft of a paper in press in Nonlinear Dynamics in Psychology and the Life Sciences, 2005

Figure 5. One-step cross prediction errors obtained using 10 sequential 1000 element segments (separate lines) to obtain predictions for all segments (abscissa) for (a) the series in Fig.1a using a 5 dimensional delay 17 embedding and a minimum neighbourhood size of 30 (nstat_z lorenz1.dat $-\# 10-\mathrm{m} 5-\mathrm{d} 17-\mathrm{k} 30-\mathrm{o}$ ) and (b) the series in Fig.1a plus Gaussian noise with double the standard deviation of the Lorenz series using a 10 dimensional, delay 1 embedding and a minimum neighbourhood size of 30 (nstat_z lorenz1. dat $-\# 10-\mathrm{m} 10-\mathrm{d} 1 \quad-\mathrm{k} 40-\mathrm{O}$ ).

Figure 6. Histograms of standardised root mean squared one-step prediction error $(\mathrm{Z})$ in a 10 dimensional delay one embedding for original (filled bar) and surrogate (unfilled bars) series (a) 50\% Noise and 1.6 standard deviation (SD) neighbourhood radius (predict n1.dat n4.dat_surr_0?? -m10 -d1 -v1.6 -o), (b) $66.6 \%$ Noise and a 1.6 SD neighbourhood radius (predict n2 . dat n4.dat_surr_0?? -m10 -d1 v1. 6 -o), and (c) $80 \%$ Noise and a 2.6 SD neighbourhood radius (predict n 4 . dat n4.dat_surr_0?? $-\mathrm{m} 10-\mathrm{d} 1-\mathrm{v} 2.6-\mathrm{o})$. (d) 14 step prediction error for the $80 \%$ noise series with a $1.6 \mathrm{SD}$ neighbourhood radius (predict $\mathrm{n} 4$. dat n4.dat_surr_0?? -m10-d1 -v1.6 -o -s14). The predict program outputs root mean squared prediction error; standardization used the mean and standard deviation of the surrogate data.

Figure 7. Root mean square locally constant prediction error as a function of prediction lag for (a) $50 \%$ and $66.6 \%$ noise series with a 1.6 standard deviation (SD) neighbourhood radius, (b) $80 \%$ noise series with a $1.6 \mathrm{SD}$ radius and a 2.6 SD radius, and (c) coarsely sampled $80 \%$ noise series, with a $1.6 \mathrm{SD}$ radius. The symbols are prediction error for the 
Penultimate Draft of a paper in press in Nonlinear Dynamics in Psychology and the Life Sciences, 2005

original series. The solid line is the mean prediction error for the 99 surrogate series. The dotted lines are a $95 \%$ confidence interval for surrogate prediction, estimated by the $3^{\text {rd }}$ and $97^{\text {th }}$ order statistics of the surrogate distribution.

Figure 8. (a) Squared correlation between the original Lorenz series from $t=2001-10000$ and filtered series, and (b) the standard deviation (SD) of the filtered series, as a function of filter iterations retaining one to three singular values (q1-q3). For the 50\% noise series, minimum neighbourhood size was either $5 \%(\mathrm{k} 400)$ or $10 \%(\mathrm{k} 800)$ of the series length, whereas for the $80 \%$ noise series it was $10 \%(\mathrm{k} 800)$ or $20 \%(\mathrm{k} 1600)$ of the series length. The standard deviation of the original Lorenz series (6.2) is indicated by a solid flat line in (b) and (c). 
Penultimate Draft of a paper in press in Nonlinear Dynamics in Psychology and the Life Sciences, 2005

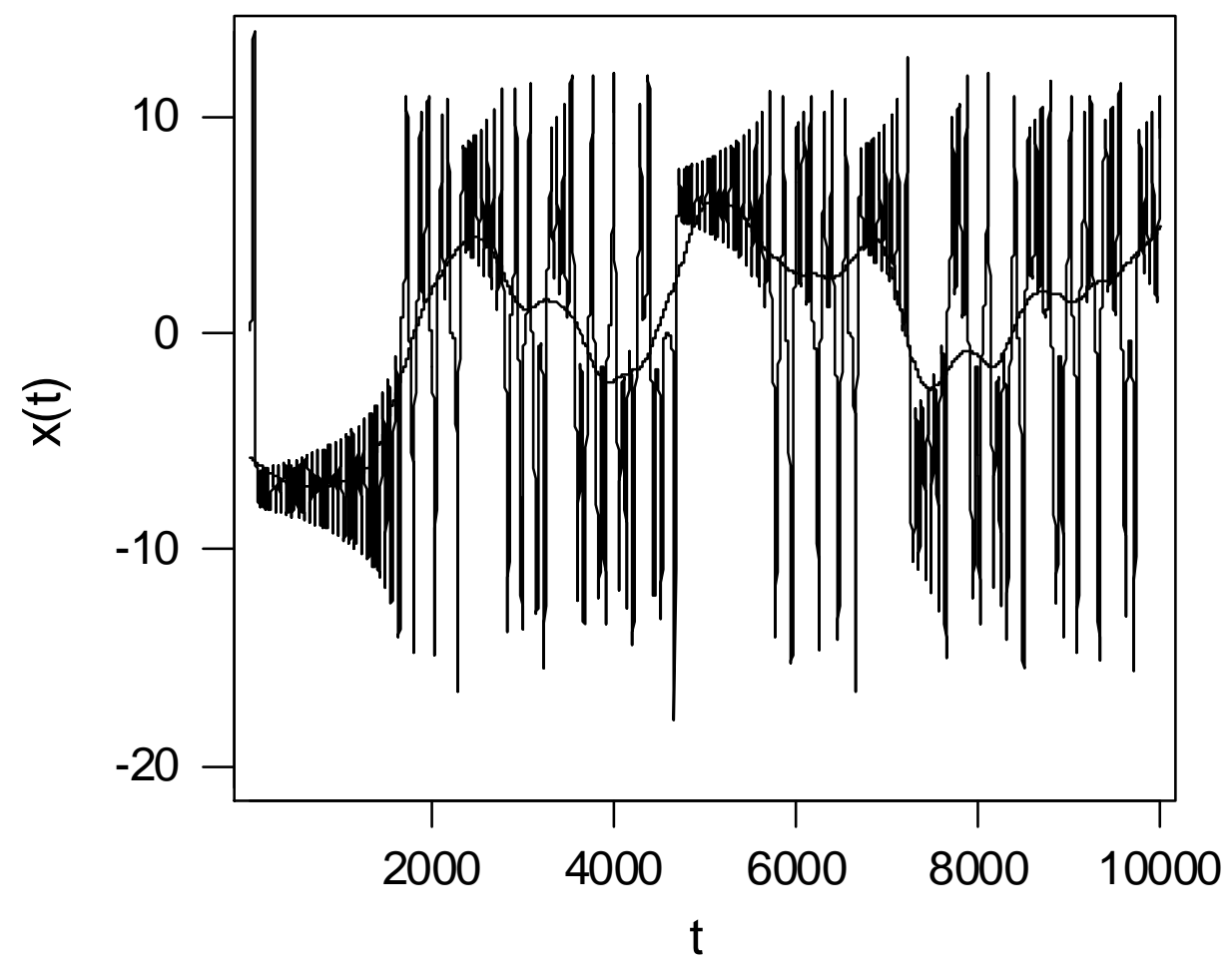

(a)

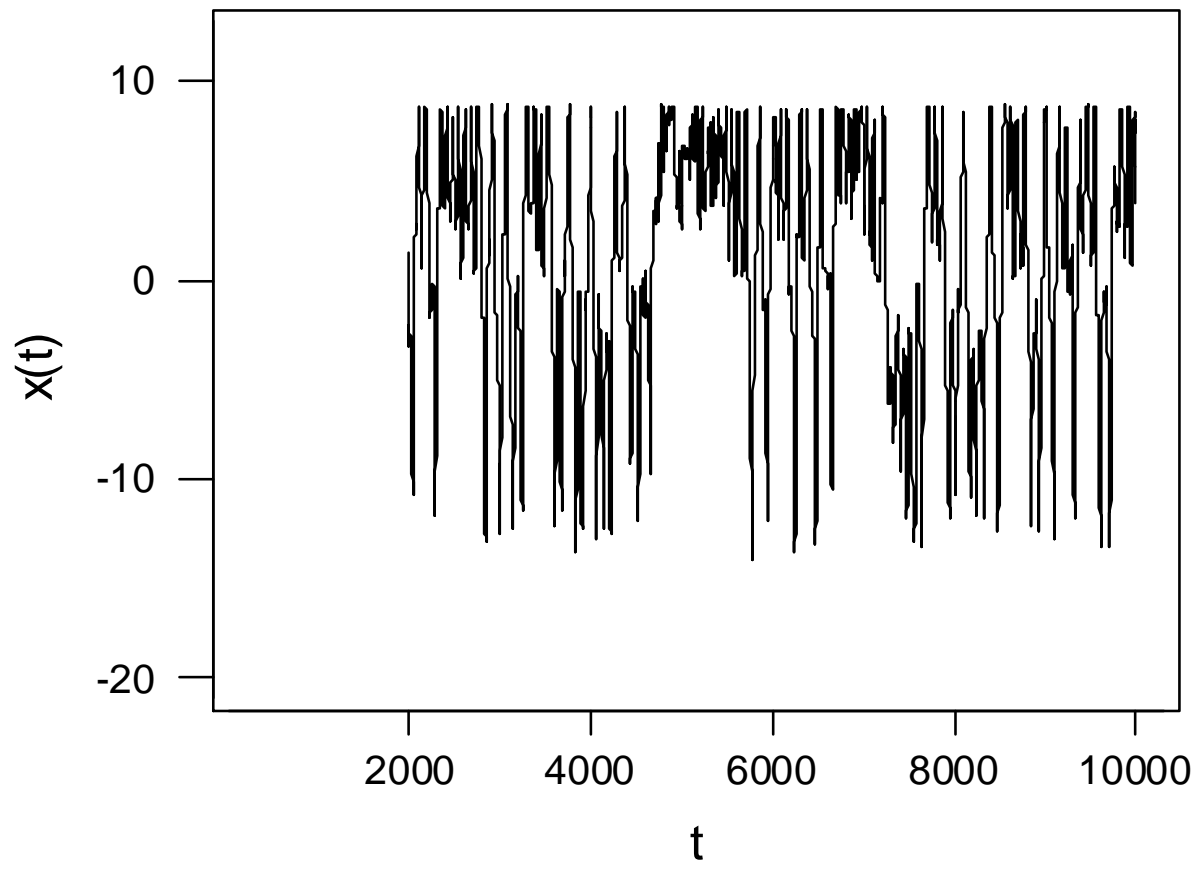

(b)

Figure 1 
Penultimate Draft of a paper in press in Nonlinear Dynamics in Psychology and the Life Sciences, 2005

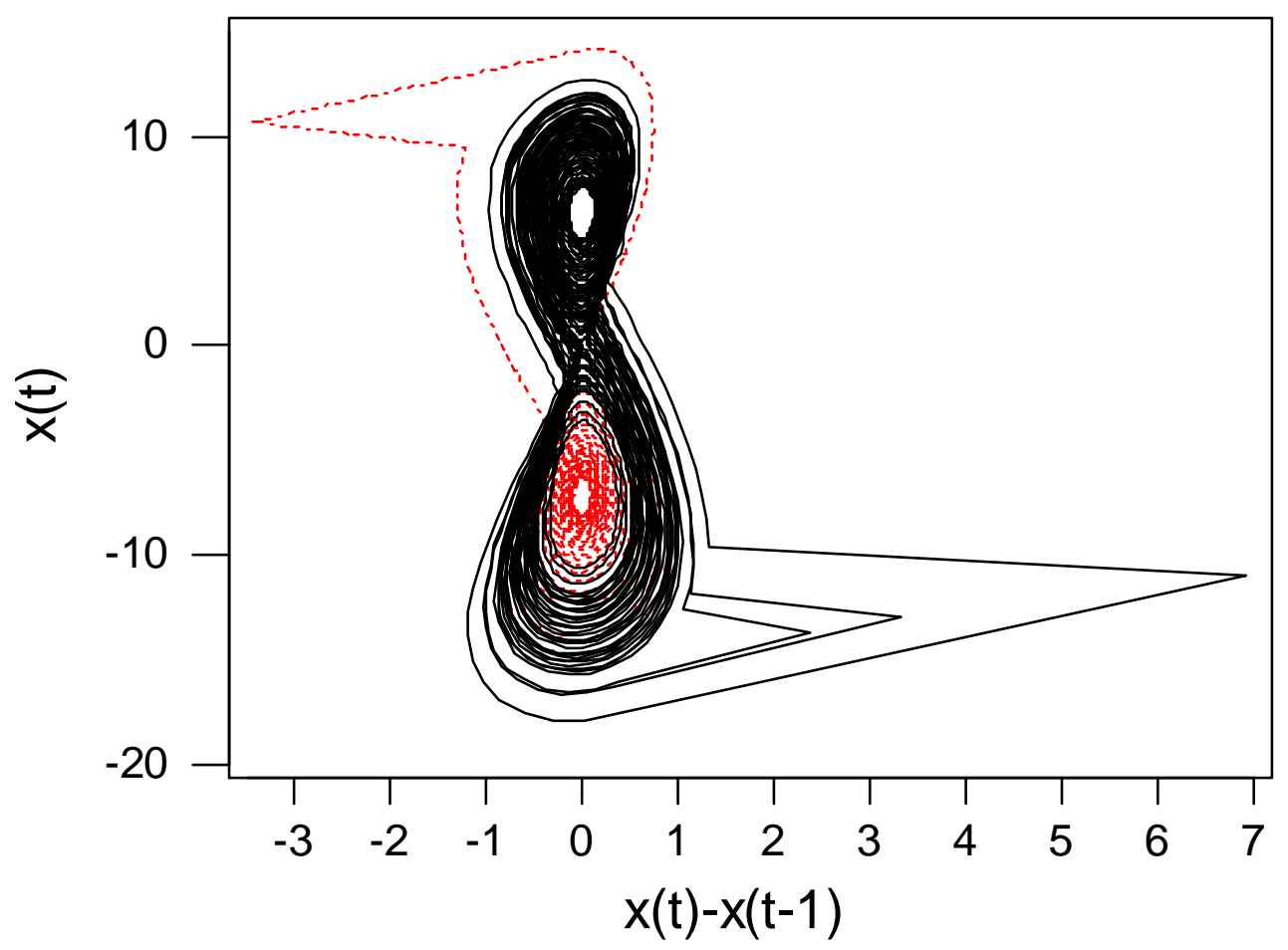

(a)

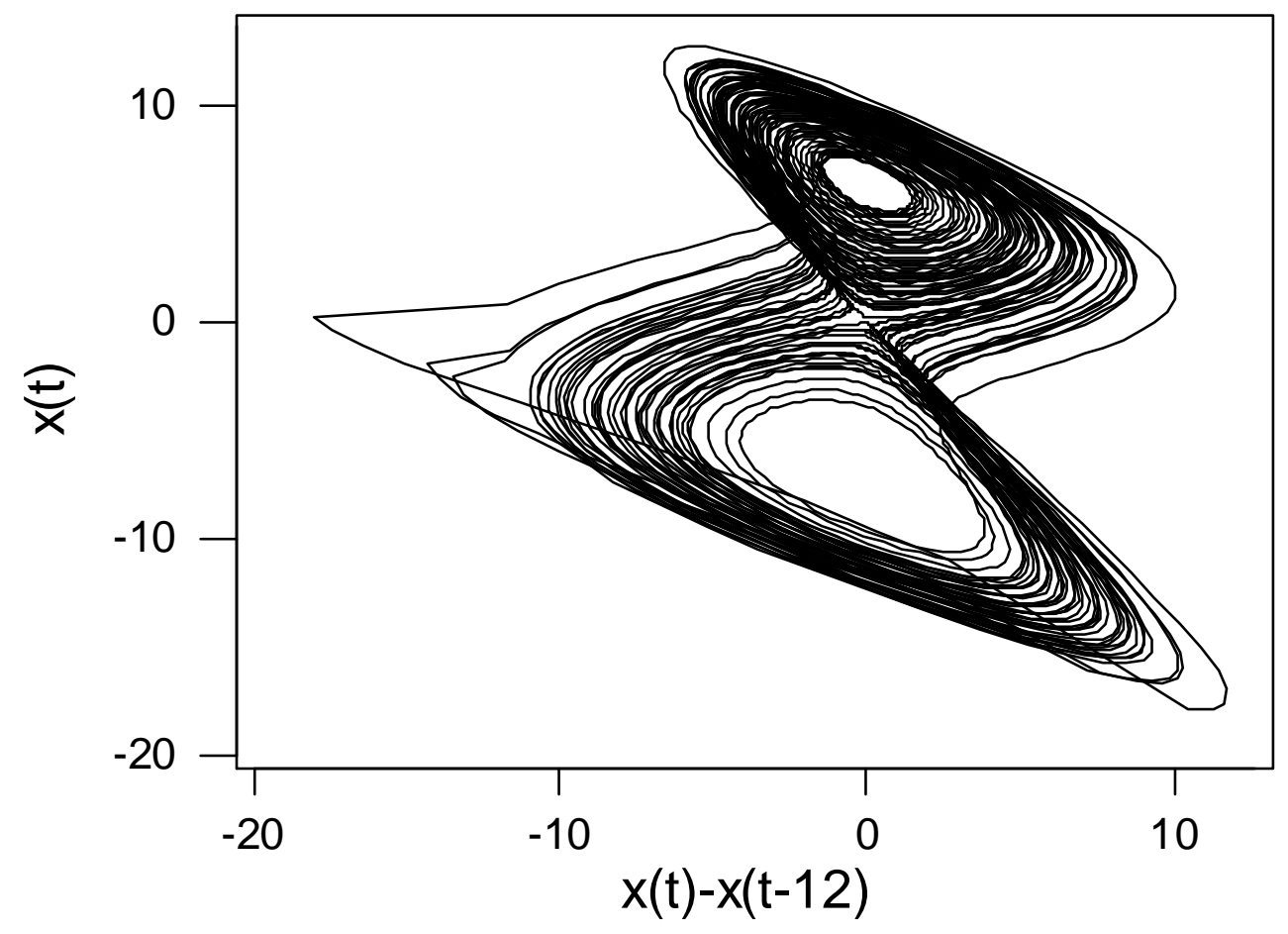

(b) 
Penultimate Draft of a paper in press in Nonlinear Dynamics in Psychology and the Life Sciences, 2005

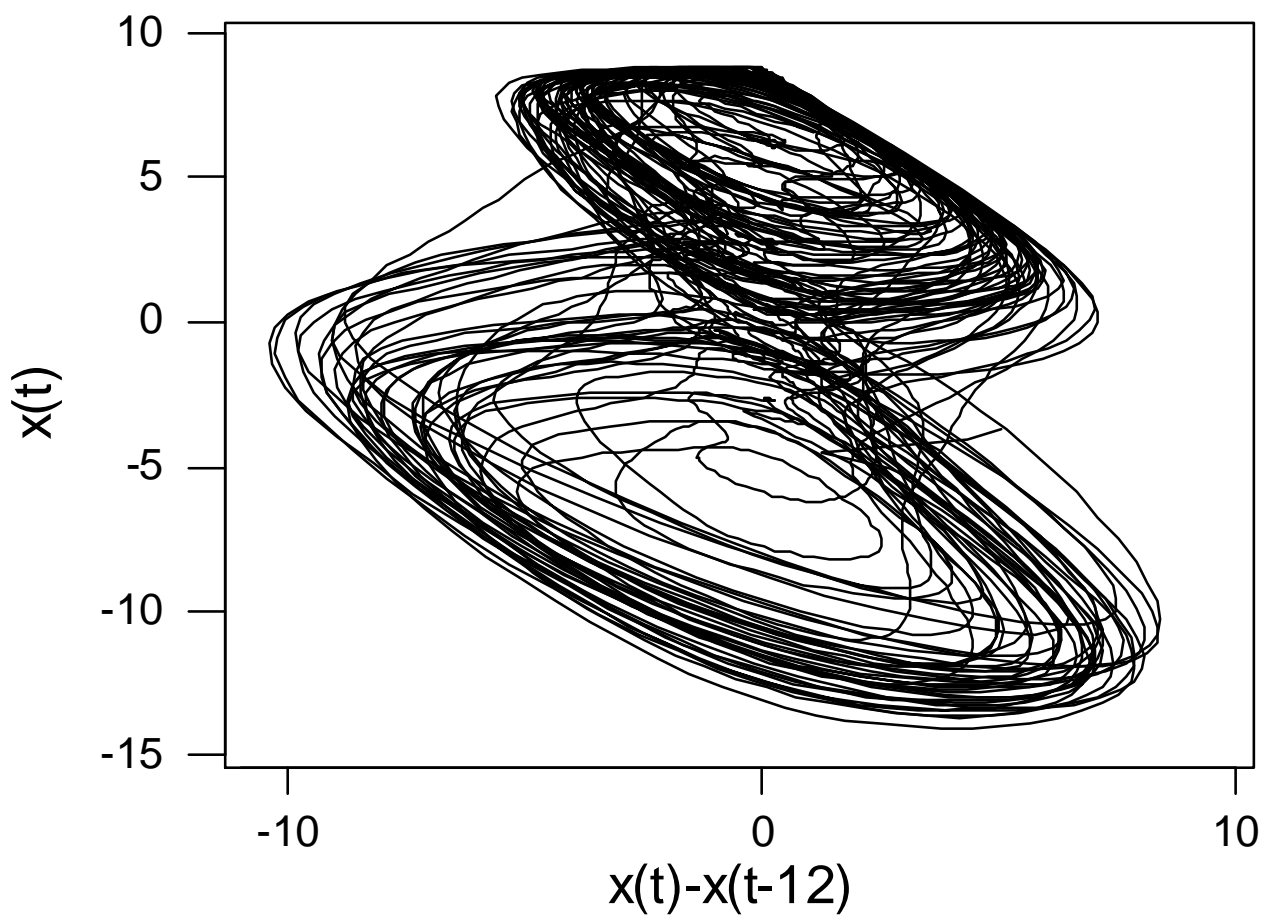

(c)

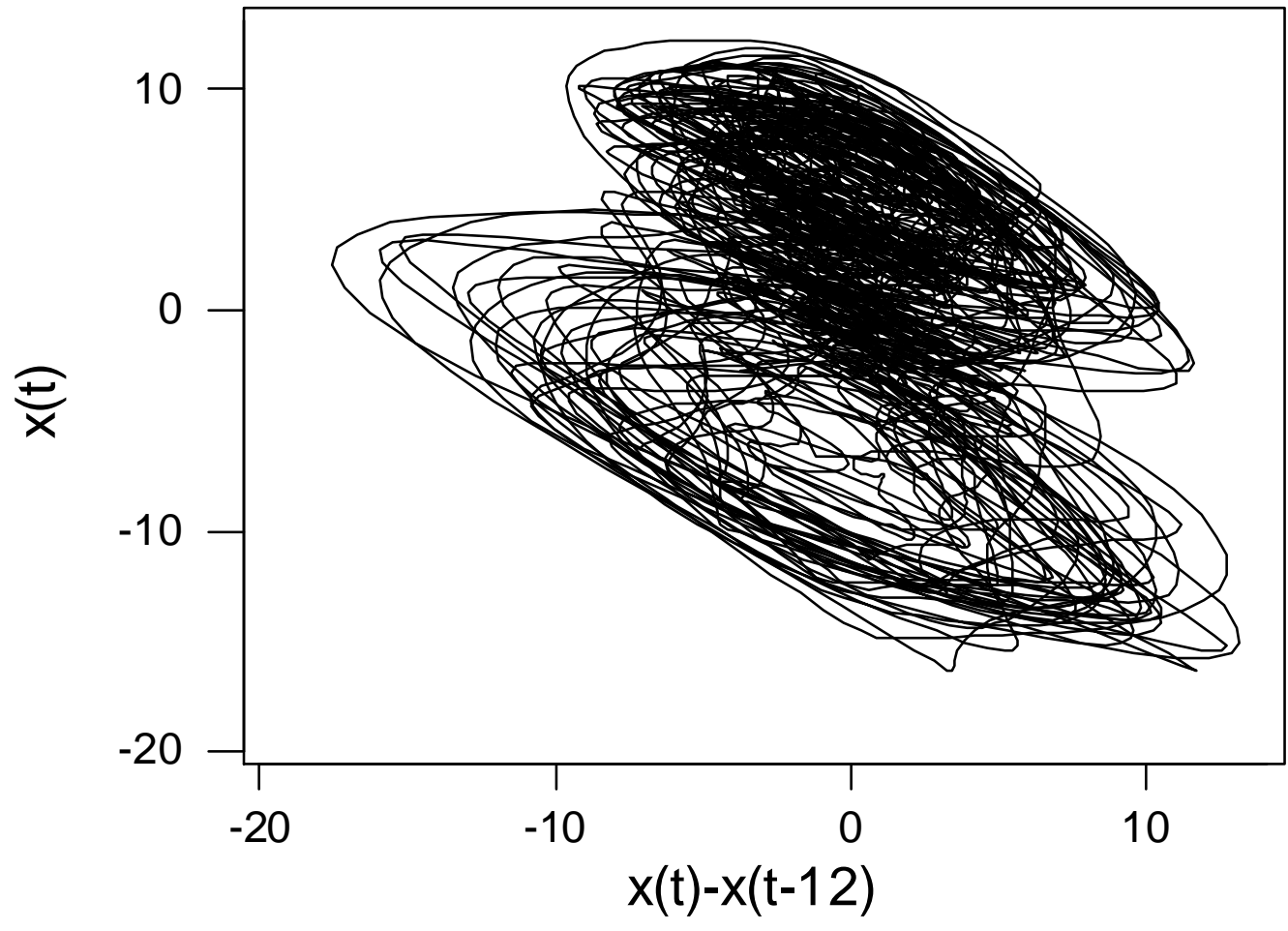

Figure 2

(d) 
Penultimate Draft of a paper in press in Nonlinear Dynamics in Psychology and the Life Sciences, 2005

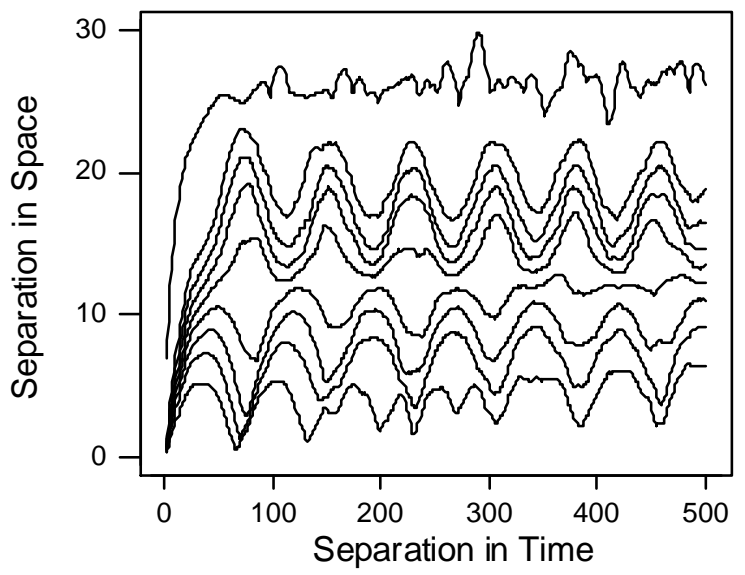

(a)

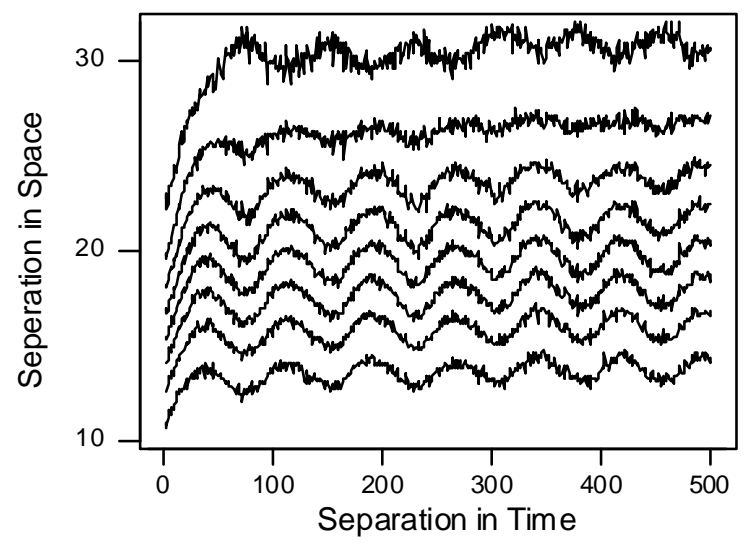

(b)

Figure 3 
Penultimate Draft of a paper in press in Nonlinear Dynamics in Psychology and the Life Sciences, 2005

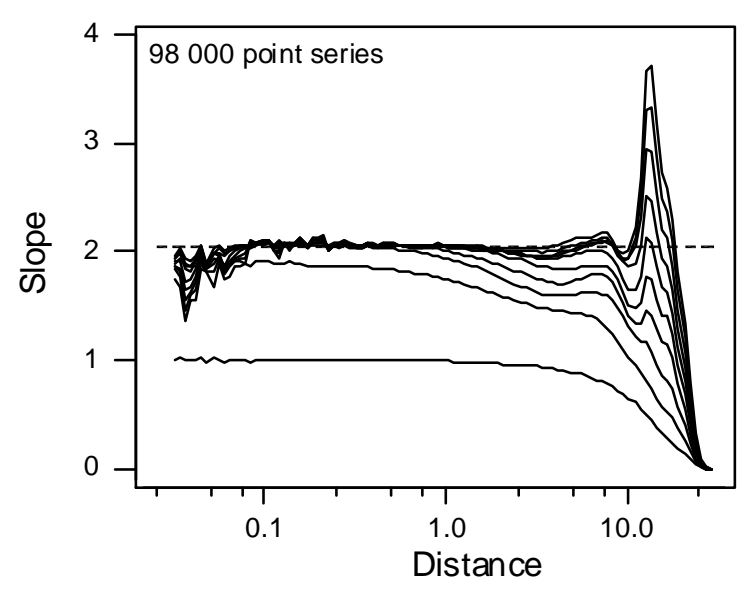

(a)

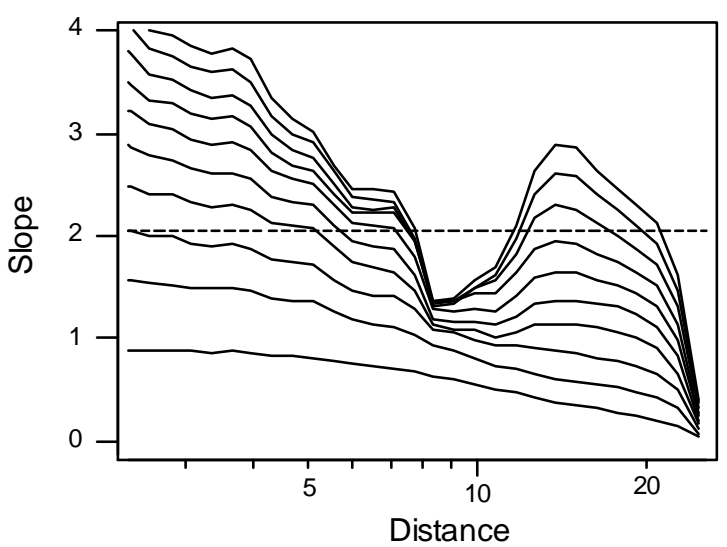

(c)

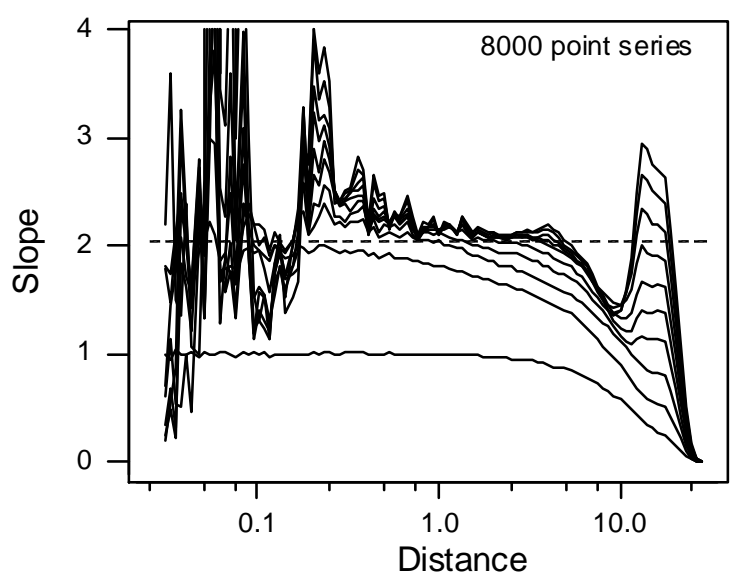

(b)

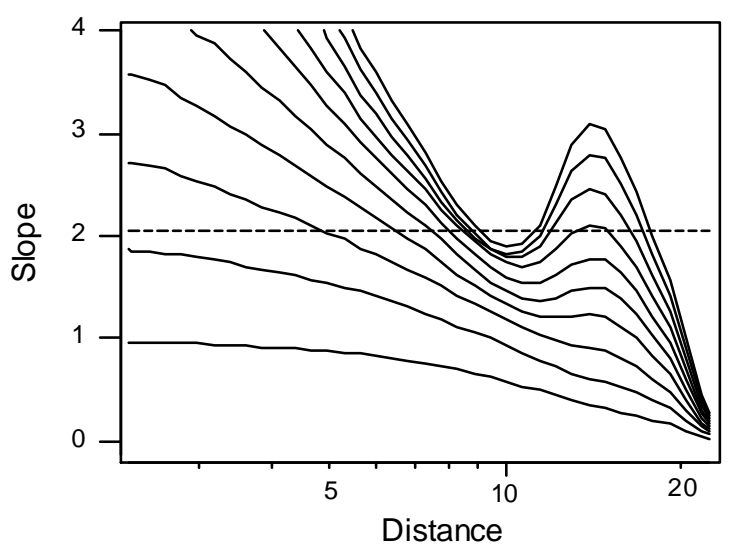

(d)

Figure 4 
Penultimate Draft of a paper in press in Nonlinear Dynamics in Psychology and the Life Sciences, 2005

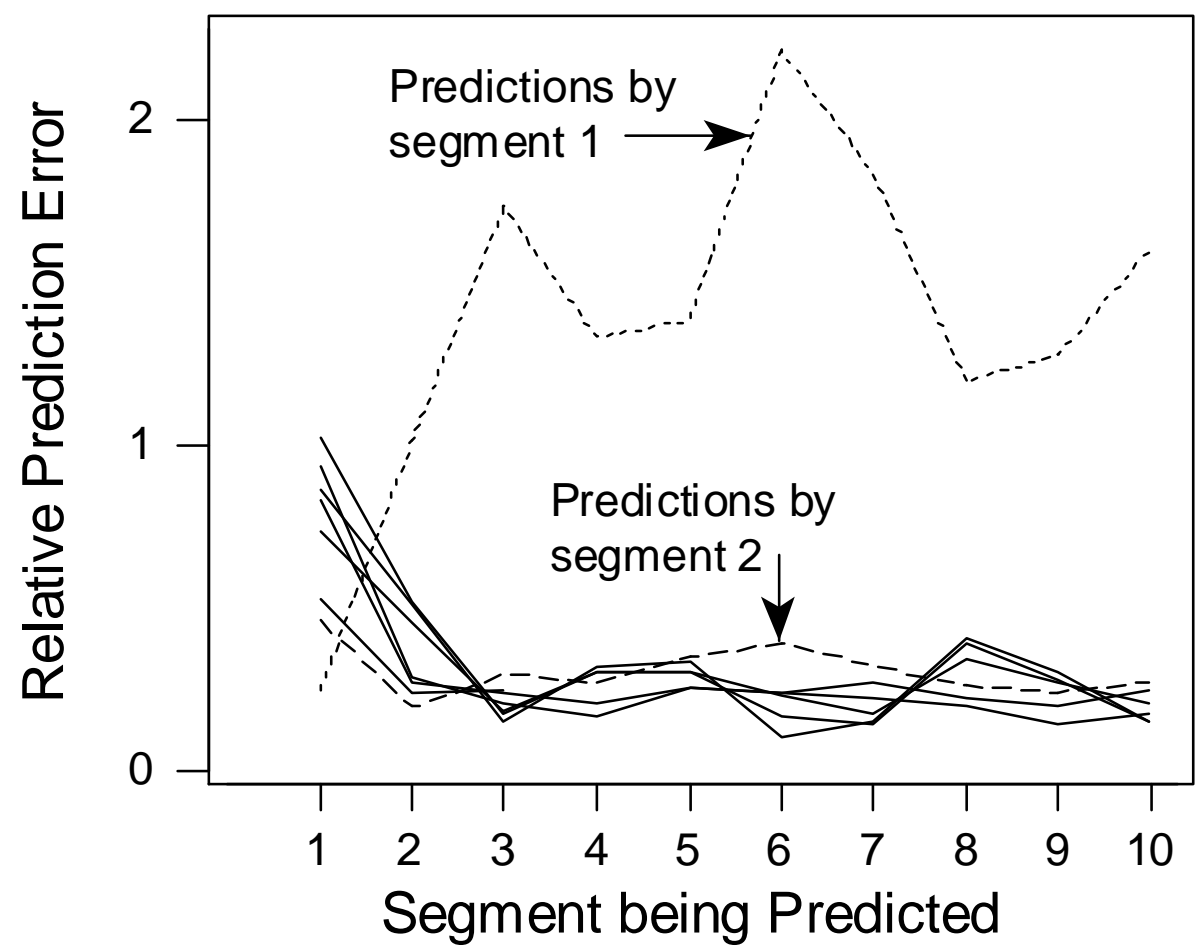

(a)

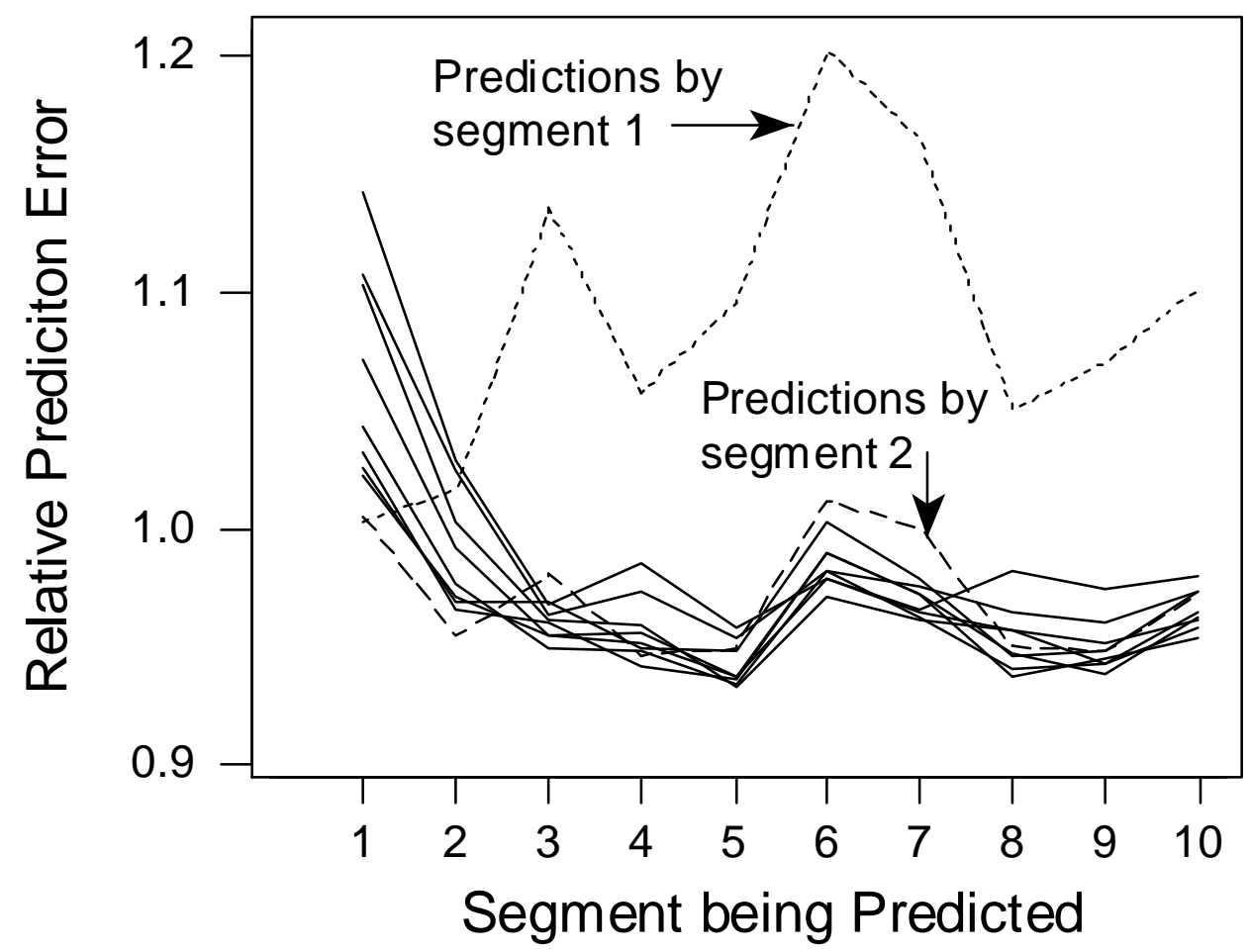

(b)

Figure 5 
Penultimate Draft of a paper in press in Nonlinear Dynamics in Psychology and the Life Sciences, 2005

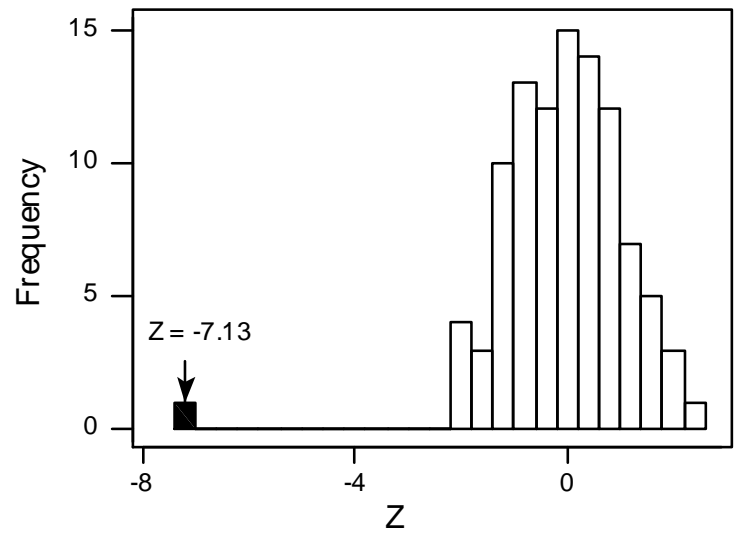

(a)

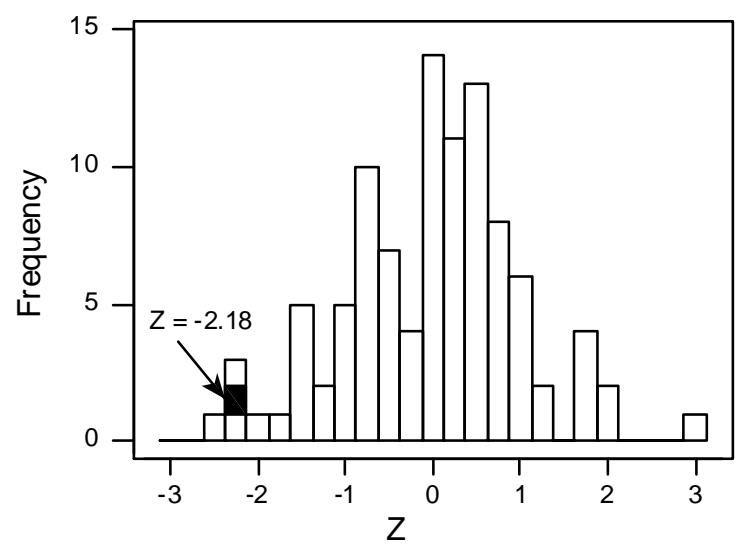

(c)

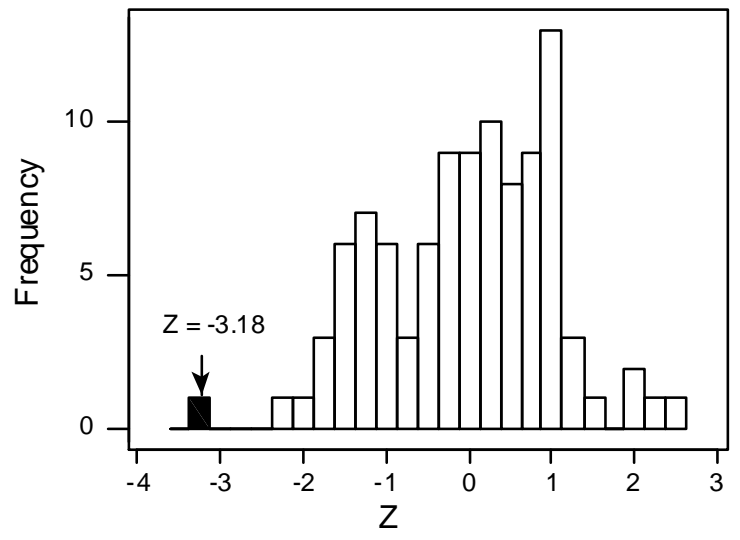

(b)

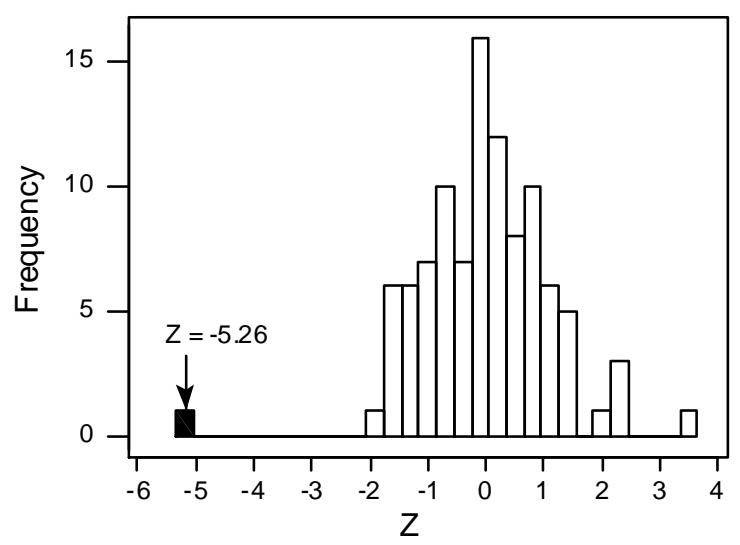

(d)

Figure 6 
Penultimate Draft of a paper in press in Nonlinear Dynamics in Psychology and the Life Sciences, 2005

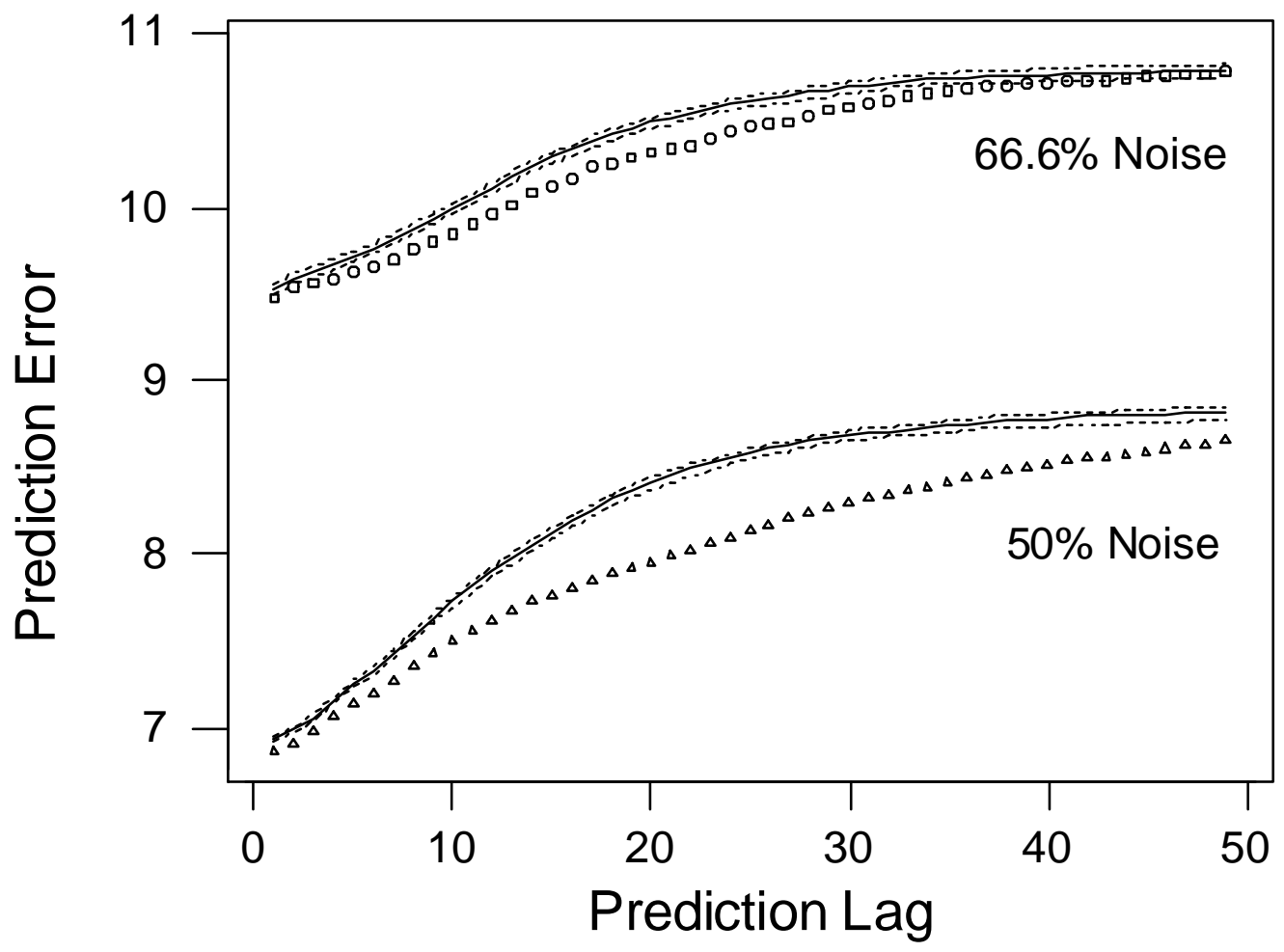

(a)

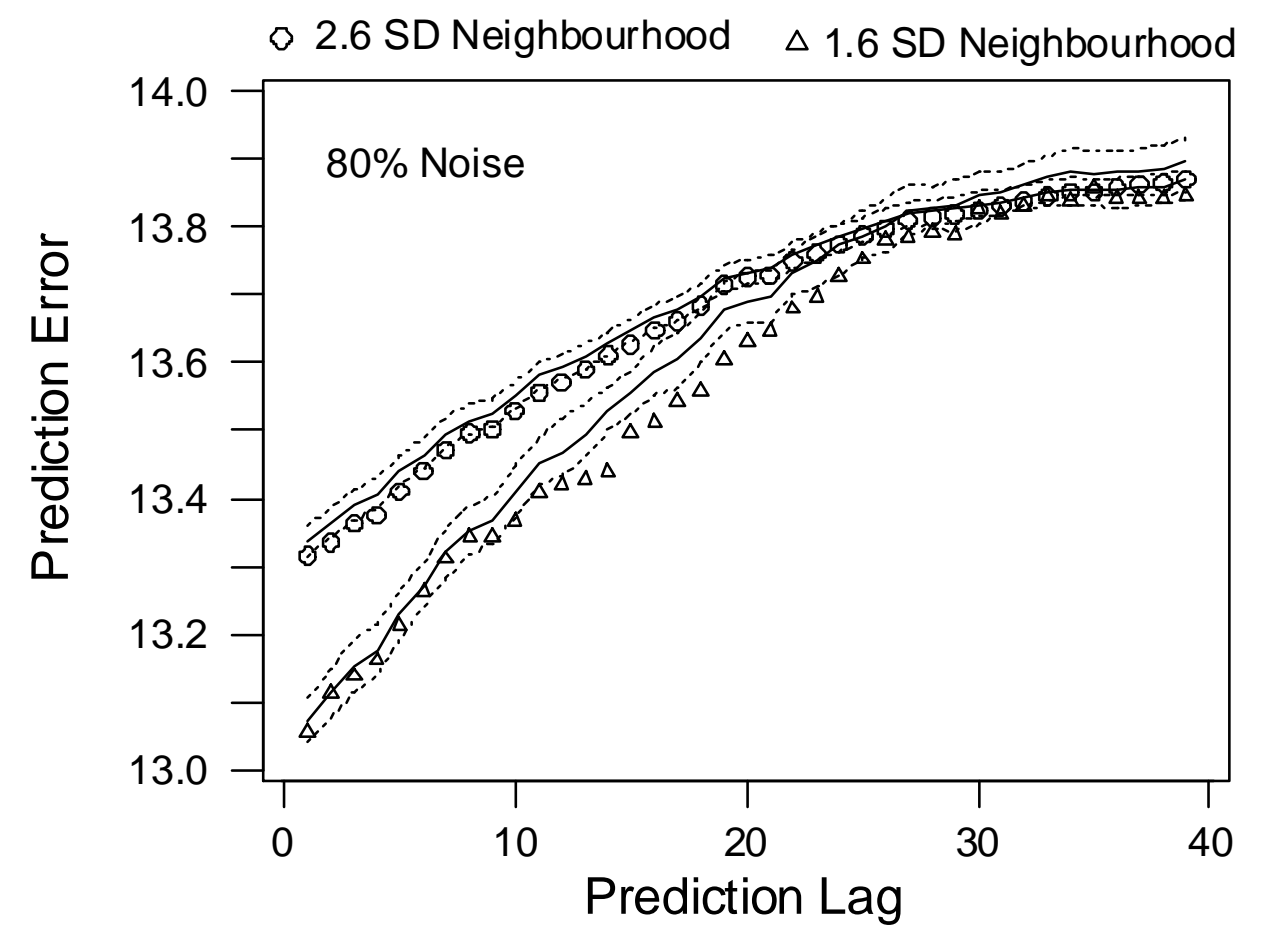

(b) 
Penultimate Draft of a paper in press in Nonlinear Dynamics in Psychology and the Life Sciences, 2005

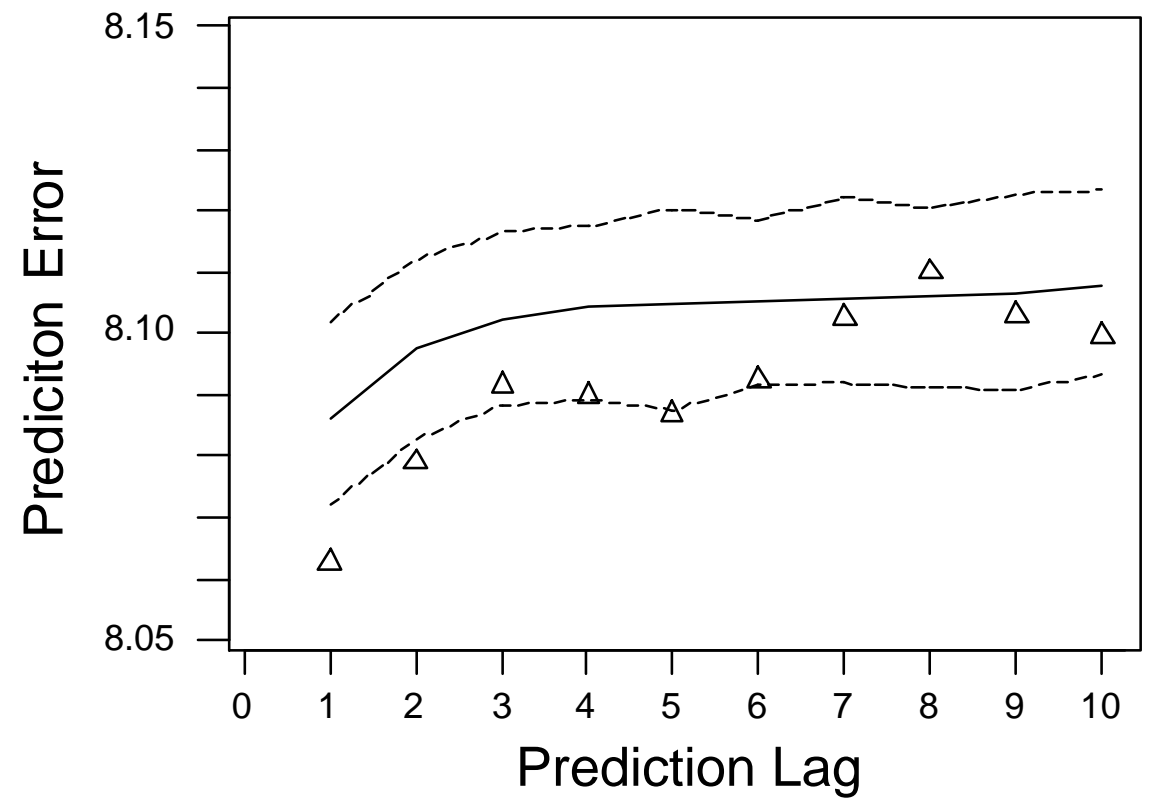

(c)

Figure 7 
Penultimate Draft of a paper in press in Nonlinear Dynamics in Psychology and the Life Sciences, 2005

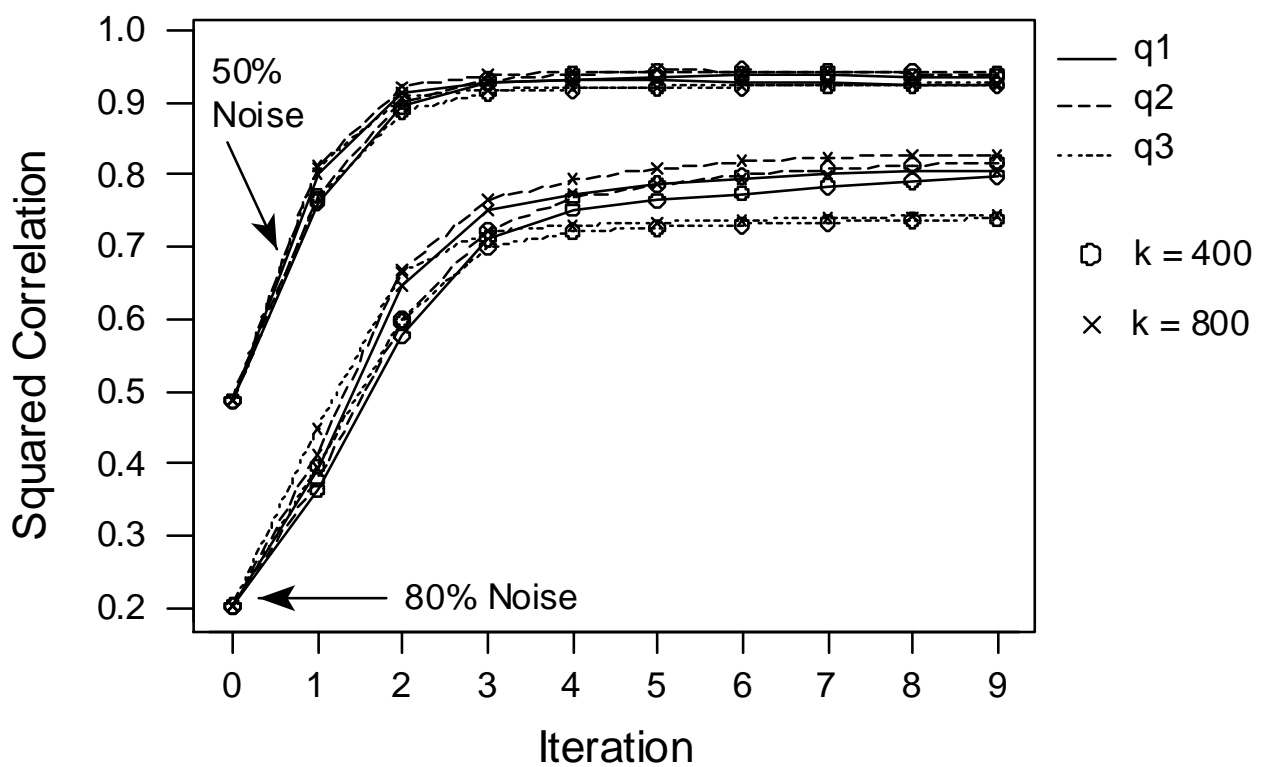

(a)

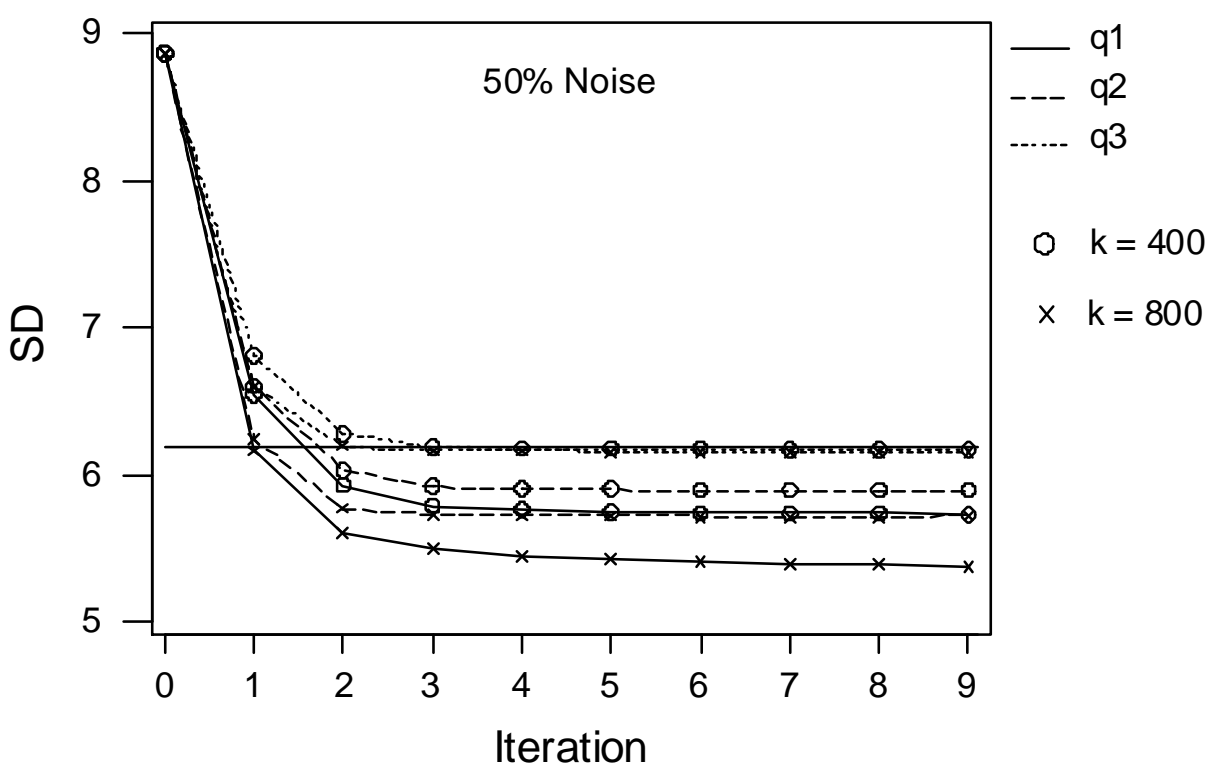

(b) 
Penultimate Draft of a paper in press in Nonlinear Dynamics in Psychology and the Life Sciences, 2005

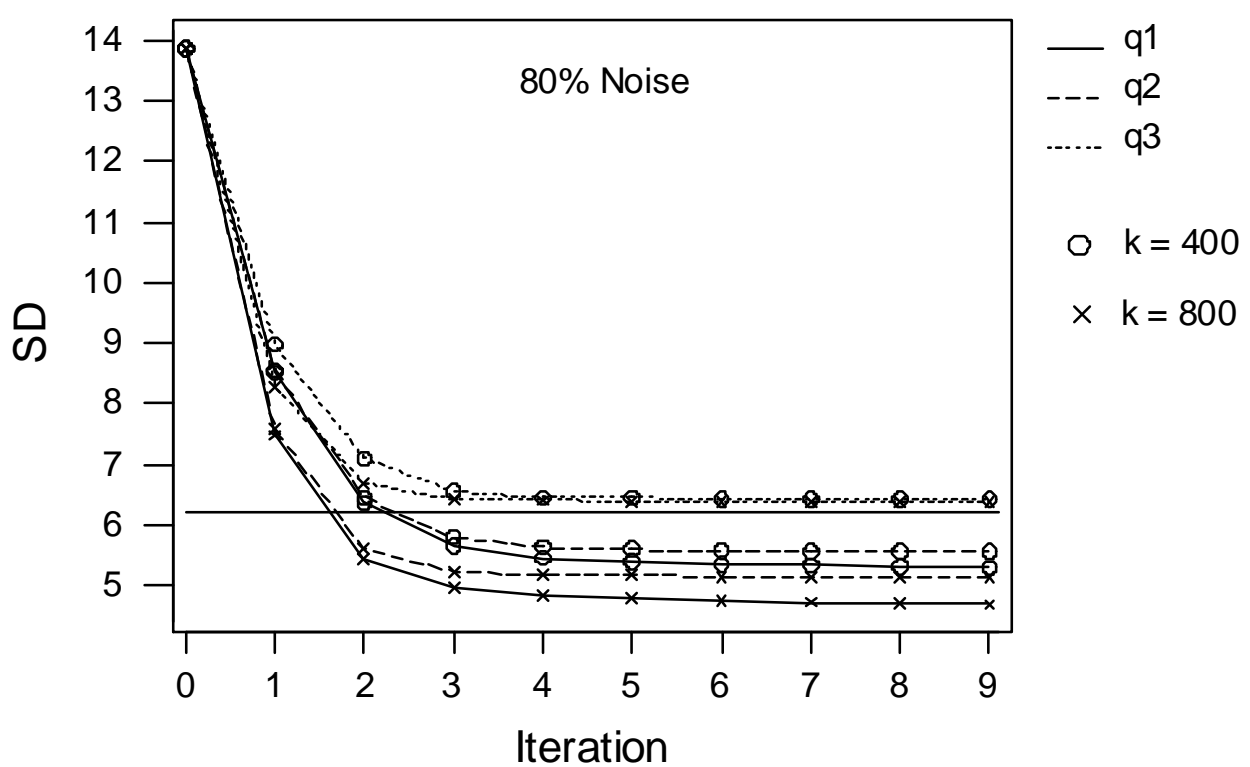

(c)

Figure 8 